\title{
Stochastic analysis of cycle slips in injection- locked oscillators and analog frequency dividers
}

\author{
Sergio Sancho, Member, IEEE, Franco Ramirez, Member, IEEE, Almudena Suarez, Fellow, IEEE
}

\begin{abstract}
A detailed investigation of cycle slips in injectionlocked oscillators and analog frequency dividers is presented. This nonlinear phenomenon gives rise to a temporal desynchronization between the injected oscillator and the input source due to noise perturbations. It involves very different time scales, so even envelope-transient based Monte Carlo analyses may suffer from high computational cost. The analysis method is based on an initial extraction of a reduced-order nonlinear model of the injected oscillator, based on harmonic-balance simulations. This model has been improved with a more accurate description of oscillation dependence on the input source either at the fundamental frequency or at a given harmonic frequency, in the case of a frequency divider. The reduced order model enables an efficient stochastic analysis of the system, based on the use of the associated Fokker-Planck equation in the phase probability density function. Several methods for the solution of the associated Fokker-Planck equation are compared, with one of them being applicable under a wider range of system specifications. The analysis enables the prediction of the parameter-space regions that are best protected against cycle slips. The technique has been applied to two microwave injectionlocked oscillators and has been validated through commercial software envelope simulations, in situations where the computational cost of the envelope simulations was acceptable, and through measurements. The measurement procedure of the cycle slipping phenomenon has been significantly improved with respect to previous work.
\end{abstract}

Index Terms - Cycle slips, injection-locked oscillators, nonlinear stochastic analysis, synchronization.

\section{INTRODUCTION}

C YCLE slipping is a statistical nonlinear phenomenon that takes place in synchronized systems under the influence of noise. During cycle slips the oscillating system becomes unlocked from the reference source, which can cause a loss of data integrity in data communication systems, reducing the ability of the system to provide communications with an acceptable error rate. In the literature, this phenomenon has been extensively analyzed in phase-locked loops (PLL) [1]-[5]. However, cycle slipping also takes place in injection-locked oscillators (ILO) [6], used at microwave frequencies for oscillator stabilization, amplification, phase

This paper was submitted for review on July 1, 2014. This paper is an expanded paper from the IEEE Int. Microwave Symposium held on June 1-6, 2014 in Tampa, Florida. This work was supported by the Spanish Ministry of Economy and Competitiveness under Contract TEC2011-29264-C03-01

Authors are with the Communications Engineering Department, University of Cantabria, Santander, 39005 Spain (e-mail: sanchosm@unican.es) shifting, quadrature generation, frequency division and other applications [7]-[21]. In the case of superharmonic ILO operating as a frequency divider [14] in a phase-locked loop, the random jumps would prevent the frequency division and give rise to an undesired phase modulation effect, disrupting the PLL response and leading to an undesired modulation of the VCO frequency. This anomalous behavior can extend over thousands of cycles of the VCO output signal. Other examples regard the injection locked active antennas, where the ILO is used to generate $\mathrm{RF} /$ microwave phase modulated signals through variation of the oscillator bias [15]-[17]. Indeed, a good number of low cost, low consume and small size transmitter and demodulators based on ILO have been presented in the literature [15]-[20]. During the random jumps the oscillator phase gets unlocked from the input signal, losing the modulation information during the time needed to recover phase-lock. This time can be of the order of thousands of reference cycles. In an ordinary application of the ILO as a local oscillator in a microwave transmitter/receiver, the oscillator phase excursion due to the phase slip would contaminate the phase of the IF output signal of the mixer, producing data loss. The ILO can also be used, for instance, for dual phase generation, achieved by means of an injection locked frequency divider by two [21] applied for quadrature modulation and quadrature down-conversion, among other. The random jumps would prevent the frequency division and disrupt the delivered signals.

The aim of this work is to investigate the impact of the cycle slipping phenomenon in injection-locked oscillators and develop a realistic and efficient methodology for its prediction at the design stage.

In an injection-locked oscillator, the self-oscillation gets synchronized to an input source, most usually corresponding to a single-tone signal at the frequency $f_{\text {in }}$, close to a given harmonic component $m f_{0}$ of the free-running oscillation. The oscillation frequency becomes commensurable with $f_{\text {in }}$ and there is a time-constant phase shift between the oscillation and the input source. The injection-locked state will be perturbed by noise associated with the input signal and by the noise sources of the oscillator circuit, giving rise to certain phase and amplitude noise spectral densities. However, due to the strong system nonlinearity in the phase variable, noise can also give rise to dynamic effects in the form of cycle slips. These take place during jumps in the oscillator perturbed phase occurring at random times. Since the phase-locking condition remains invariant under these jumps in the oscillator 
phase, the system will recover phase lock after each jump. However, during the jumps, the oscillator is unlocked from the injection source, with the number of reference cycles slipped being determined by the transition time length.

Since the occurrence of two jumps in a single stochastic realization can be separated in time by many thousands of reference cycles, the computational cost, even with envelope transient-based Monte Carlo analyses, would generally be very high. In the case of PLLs, this problem was solved in the seminal works [1]-[2] with the Fokker-Planck equation, used to predict the cycle slip probability by means of eigenfunction expansion methods [24]. In first and second-order PLL systems, the practical analysis [1]-[2] was very efficient thanks to the reduced number $n$ of state variables involved (usually $n<3$ ). In the case of free-running oscillators, the stochastic characterization of the oscillator phase variable has also been performed in previous works [25]-[27]. Although these systems do not present coexisting synchronized solutions and therefore no random jumps are produced, the stochastic techniques proposed in these works have influenced the present analysis.

In the case of the microwave injection-locked oscillators, a Fokker-Planck equation directly obtained from the differential equation system is unmanageable in practice, due to the high number of state variables involved. To circumvent this problem, here the Fokker-Planck equation associated with the phase variable will be derived from a reduced-order semianalytical model of the injected oscillator. The reduced-order differential equation system is obtained from magnitudes extracted from Harmonic Balance (HB) simulations. This model is similar to the one used in [12]-[22] to describe the deterministic transient dynamics of an injected oscillator. However, the model will be improved here with a more accurate description of oscillator dependence on the input source, which will increase the model reliability and applicability. Unlike previous works [12]-[22], here a stochastic analysis will be carried out. A nonlinear stochastic differential equation will be derived, able to predict nonlinear phenomena associated with noise perturbations.

The reduced order of the semi-analytical differential equations will enable the extraction of a simple Fokker-Planck equation. Note that the structure of this equation is different from the Fokker-Planck equation associated with the phase deviation in a free-running oscillator [26]. On the one hand, it corresponds to an injection-locked oscillator and not to a freerunning one. On the other hand, the resolution procedure will be particularized in order to stochastically characterize the jumps between adjacent synchronized solutions.

This equation will be analyzed in terms of eigenfunctions and eigenvalues of the Fokker-Planck operator. The technique permits an efficient analysis of the oscillator circuit performance in the presence of stochastic signals. It allows the oscillator transient dynamics to be studied under noise perturbations and can be efficiently solved to predict the probability of the phase jumps. The proposed methodology allows and an insightful study of the influence of the circuit parameters on the cycle slipping phenomenon.
Two major new contributions with respect to the previous work [23] are the modification of the semi-analytical model to increase accuracy and broaden applicability and its extension to enable the analysis of cycle slips frequency dividers. In the present work, several methods for the solution of the FokkerPlanck equation are provided. A comparison of various analysis methods is presented, with one of these methods being applicable under a wider range of specifications of the system, unlike the situation in [15]. Finally, the measurement procedure of the cycle slipping phenomenon has also been improved, allowing the measurement of time-domain realizations containing random jumps. In this manner it will be possible to characterize experimentally the mean time between phase jumps.

The paper is organized as follows. Section II introduces the analytical formulation for the analysis of fundamentallysynchronized oscillators and analog frequency dividers both in the steady state and noise-perturbed regimes. The obtained semi-analytical model is proved to explain and predict the cycle slipping phenomenon. In Section III the Fokker-Planck equation associated with the semi-analytical model is solved following the methods proposed in [1],[24]. The resulting expressions are applied to analyze the influence of the circuit parameters on the probability of random jumps. In Section IV, the comparison of the technique predictions with experimental results in an injection-locked oscillator at $1 \mathrm{GHz}$ is provided.

\section{SEMI-ANALYTICAL MODEL}

\section{A. Steady-state analysis}

The system composed of an oscillator synchronized with an external source will be called in general injection-locked oscillator (ILO). Depending on the harmonic relationship between the oscillator free-running frequency and the external source, the system can be a fundamentally-synchronized oscillator or a frequency divider. In the absence of the synchronizing source, the free-running oscillator frequency will be denoted by $\omega_{0}=2 \pi f_{0}$. Without loss of generality, we will assume that the synchronizing source (reference) is a small-amplitude single-tone voltage generator:

$$
v_{i n}(t)=U_{-m} e^{-j \omega_{i n} t}+U_{m} e^{j \omega_{i n} t}, \quad U_{m}=U e^{j \phi_{i n}}, \quad U \in \mathbb{R}
$$

with $\omega_{\text {in }}=m\left(\omega_{0}+\Delta \omega\right)$. The parameter $m \in \mathbb{Z}^{+}$has been introduced to include the case of a frequency divider, whereas $\Delta \omega$ is assumed to be small enough to maintain the input frequency $\omega_{\text {in }}=2 \pi f_{\text {in }}$ within the ILO synchronization range. In the synchronized state, the steady state solution is periodic with the fundamental frequency $\omega=\omega_{i n} / m$, where $m=1$ in the case of fundamentally synchronized oscillator. For better insight into the reduced-order model derivation, the circuit level harmonic balance formulation of the ILO will be considered first, with $n$ state variables and $N$ harmonic terms. Using the modified nodal harmonic-balance formulation, this can be expressed in matrix form as [12]: 


$$
\bar{F}(\bar{X})+[j \omega] \bar{Q}(\bar{X})+H(j \omega) \bar{X}+\bar{G}=\overline{0}
$$

where $\bar{X}, \bar{F}, \bar{Q}$ and $\bar{G}$ are the vectors containing the harmonic components of the node voltages and inductor currents $\bar{x}(t)$, the harmonic terms of resistive currents and loop voltages $\bar{f}(t)$, the harmonics of the linear and nonlinear charges and fluxes $\bar{q}(t)$, and the independent input sources, respectively. Matrix $H$ contains the transfer functions of the distributed elements.

An auxiliary generator (AG) [12]-[22] of voltage type will be used here for the ILO analysis. The AG operates at the fundamental frequency $\omega=\omega_{\text {in }} / m$ with an amplitude $V_{1}$ and phase $\phi_{1}$. It must fulfill a non-perturbation condition, corresponding to the zero value of the AG current-to-voltage ratio, that is [12]-[22]:

$$
Y_{p}=\frac{I_{1}^{p}}{V_{1} e^{j \phi_{1}}}=0
$$

where $p$ is the analysis node at which the AG is connected. Then, the vector $\bar{G}$ in (2) will contain, together with the bias sources, the input periodic source and the AG, which behaves in system (2) as an independent source. Using (2) as an inner tier to solve $\bar{X}$ in terms of the independent sources, the equation associated with current $I_{1}^{p}$ can be expressed as:

$$
I_{1}^{p}\left(V_{1}, \phi_{1}, U_{m}, U_{-m}, \omega\right)=0
$$

where the dependence of $I_{1}^{p}$ on $U_{-m}$ is indicated explicitly. Actually, the nonlinearities depend on the full set of harmonic components $\bar{X}_{k}$ of the state variables, where $k$ goes from $-N$ to $N$. The vector $\bar{X}_{-m}$ will be affected by $U_{-m}$ through the linear embedding network and this dependence will, in general, be transferred to $I_{1}^{p}$. The dependence on $U_{-m}$, which had not been considered in previous works [12]-[23], will be taken into account when developing the model of the ILO. Equation (4) can be expressed in terms of the admittance function as:

$$
I_{1}^{p}\left(V_{1}, \phi_{1}, U_{-m}, U_{m}, \omega\right)=Y_{p}\left(V_{1}, \phi_{1}, U_{-m}, U_{m}, \omega\right) V_{1} e^{j \phi_{1}}=0
$$

where $Y_{p}$ is the first harmonic total admittance at node $p$. Provided the input source amplitude is small enough, (5) can be approached by a first-order Taylor series about the freerunning values of the voltage and frequency variables as:

$$
\begin{array}{r}
Y_{p}\left(V_{1}^{0}+\Delta V, \phi_{1}, U_{-m}, U_{m}, \omega_{0}+\Delta \omega\right) \simeq \\
\simeq Y_{V} \Delta V+Y_{\omega} \Delta \omega+B_{m} U_{m}+B_{-m} U_{-m}=0, \\
Y_{V}=\frac{\partial Y_{p}\left(V_{1}^{0}, \phi_{1}, 0,0, \omega_{0}\right)}{\partial V_{1}}, \quad Y_{\omega}=\frac{\partial Y_{p}\left(V_{1}^{0}, \phi_{1}, 0,0, \omega_{0}\right)}{\partial \omega}, \\
B_{m}=\frac{\partial Y_{p}\left(V_{1}^{0}, \phi_{1}, 0,0, \omega_{0}\right)}{\partial U_{m}}, \quad B_{-m}=\frac{\partial Y_{p}\left(V_{1}^{0}, \phi_{1}, 0,0, \omega_{0}\right)}{\partial U_{-m}}
\end{array}
$$

Equation (10) can be applied to obtain the ILO
synchronization range. For this purpose, it is more convenient

Equation (10) can be applied to obtain the ILO
synchronization range. For this purpose, it is more convenient to use the derivatives:

where $V_{1}^{0}$ is the first harmonic amplitude at the analysis node $p$ under free-running conditions, $B_{-m}, B_{m}$ are injection sensitivity terms and $\Delta \omega=\omega_{i n} / m-\omega_{0}$ is the frequency shift. Equation (6) constitutes a semi-analytical model of the ILO, where the admittance derivatives can be numerically obtained through finite differences, introducing an auxiliary generator in commercial Harmonic Balance software as explained in [12]-[23]. In these previous works, the term $B_{-m}$ was not considered, which reduced the validity of the model to those analysis nodes where this term vanishes. At those nodes the implicit dependence of the current $I_{1}^{p}$ on $U_{-m}$ must be negligible or zero. This condition is usually difficult to attain when the input generator and the analysis node are placed at different sides of an active element, such as a transistor, which reduces the applicability of the model.

In the following, the effect of the inclusion of the term $B_{-m}$ in the semi-analytical model will be analyzed. First, (6) will be rewritten explicitly showing the dependence on the input generator phase:

$$
\begin{aligned}
& Y_{p}\left(V_{1}, \phi_{1}, U, \phi_{i n}, \omega\right)= \\
& \quad=Y_{V} \Delta V+Y_{\omega} \Delta \omega+B_{m} U e^{j \phi_{i n}}+B_{-m} U e^{-j \phi_{i n}}=0
\end{aligned}
$$

In the synchronized state, the steady-state condition (7) must be preserved under an arbitrary time shift in the whole system of state variables, including the input source:

$$
\begin{aligned}
0 & =Y_{p}\left(V_{1}, \phi_{1}+\alpha, U, \phi_{i n}+m \alpha, \omega\right)= \\
& =Y_{V} \Delta V+Y_{\omega} \Delta \omega+B_{m} U e^{j\left(\phi_{i n}+m \alpha\right)}+B_{-m} U e^{-j\left(\phi_{i n}+m \alpha\right)}, \forall \alpha \in \mathbb{R}
\end{aligned}
$$

In order for (8) to be fulfilled for any phase shift value $\alpha$, the coefficients $B_{-m}$ and $B_{m}$ must be phase-dependent with the form:

$$
\begin{aligned}
& \frac{\partial Y_{p}\left(V_{1}, \phi_{1}+\alpha, U, \phi_{i n}+m \alpha, \omega\right)}{\partial \alpha}=0, \quad \forall \alpha \rightarrow \\
& \rightarrow B_{m}\left(\phi_{1}\right)=B_{m}^{0} e^{-j m \phi_{1}}, \quad B_{-m}\left(\phi_{1}\right)=B_{-m}^{0} e^{j m \phi_{1}}
\end{aligned}
$$

where $B_{m}^{0}, B_{-m}^{0}$ are complex numbers depending on the circuit topology and the free-running solution. The components $Y_{V}$, $Y_{\omega}$ are phase independent. In previous works [12]-[23], the phase dependence (9) was not derived. Here, as will be shown in the Section II.B, the expression of $B_{-m}$ and $B_{m}$ as phasedependent functions will provide a powerful formulation for analyzing the ILO dynamics in the presence of noise sources. Introducing expressions (9) in (7), the steady-state semianalytical model is obtained:

$$
Y_{V} \Delta V+Y_{\omega} \Delta \omega+B_{m}^{0} U e^{j\left(\phi_{\phi_{i n}}-m \phi_{1}\right)}+B_{-m}^{0} U e^{-j\left(\phi_{m}-m \phi_{1}\right)}=0
$$
the derivatives: 


$$
\begin{aligned}
Q_{R} & \equiv \frac{\partial Y_{p}}{\partial U_{m}^{r}}=\frac{\partial Y_{p}}{\partial U_{m}} \frac{\partial U_{m}}{\partial U_{m}^{r}}+\frac{\partial Y_{p}}{\partial U_{-m}} \frac{\partial U_{-m}}{\partial U_{m}^{r}}=B_{m}^{0} e^{-j m \phi_{1}}+B_{-m}^{0} e^{j m \phi_{1}} \\
Q_{I} & \equiv \frac{\partial Y_{p}}{\partial U_{m}^{i}}=\frac{\partial Y_{p}}{\partial U_{m}} \frac{\partial U_{m}}{\partial U_{m}^{i}}+\frac{\partial Y_{p}}{\partial U_{-m}} \frac{\partial U_{-m}}{\partial U_{m}^{i}}=j B_{m}^{0} e^{-j m \phi_{1}}-j B_{-m}^{0} e^{j m \phi_{1}}
\end{aligned}
$$

By substituting $B_{-m}$ and $B_{m}$ in terms of $Q_{r}$ and $Q_{i}$ into (11), we arrive to:

$$
\begin{aligned}
& Y_{V} \Delta V+Y_{\omega} \Delta \omega+Q_{R} U \cos m \varphi+Q_{I} U \sin m \varphi=0, \\
& \varphi \equiv \frac{\phi_{\text {in }}}{m}-\phi_{1}
\end{aligned}
$$

Complex equation (12) leads to the following expression for the frequency shift:

$$
\Delta \omega=U \frac{Q_{R} \times Y_{V} \cos m \varphi+Q_{I} \times Y_{V} \sin m \varphi}{Y_{V} \times Y_{\omega}}
$$

In (13) the following simplifying relation has been applied: $a \times b=a^{r} b^{i}-a^{i} b^{r}$, where the super-indexes $(r, i)$ mean real and imaginary parts. Equation (13) provides the frequency shift variation as a function of the phase difference $\varphi$. Equation (13) describes a periodic frequency shift variation in the interval $\varphi \in[0,2 \pi / \mathrm{m}]$. The synchronization frequency limits can be obtained by numerically calculating the maximum and minimum values of function (13) in that interval.

Note that, in the particular case $B_{-m}=0$, the admittance function at the analysis node fulfills:

$$
\begin{aligned}
& Y_{p}\left(V_{1}, \phi_{1}, U, \phi_{i n}, \omega\right)=Y_{p}\left(V_{1}, \phi_{1}, U e^{j \phi_{\text {in }}}, \omega\right) \rightarrow \\
& \quad \rightarrow Q_{R}^{r}=Q_{I}^{i}, \quad Q_{I}^{r}=-Q_{R}^{i}
\end{aligned}
$$

where the super-indexes $(r, i)$ mean real and imaginary parts. Equations (14) show that the Cauchy-Riemann equations associated with the first harmonic $U_{1}$ of the voltage source are fulfilled. In previous works [12]-[23], only analysis nodes fulfilling (14) were considered. Formulation (10) allows the semi-analytical model to be extended to the general case in which the term $B_{-m}$ does not vanish at the analysis node.

As an example, the term $B_{-m}$ has been calculated at two different nodes in the FET-based injection-locked oscillator of Fig. 1. The circuit has been simulated in commercial software. The case $m=1$ has been selected. Both terms have been represented in Fig. 2 as the phase $\phi_{1}$ is varied in the range $[0,2 \pi]$. As can be seen in the figure, for the analysis node $p_{1}$ situated at the transistor gate we find $B_{-1} \simeq 0$. On the other hand, for the analysis node $p_{2}$ (transistor drain) the term $B_{-1}$ does not vanish and fulfills relation (9). When selecting this node for the analysis, the term $B_{-1}$ must be taken into account in the semi-analytical model. Note that, in this case, the transistor device is in the circuit path connecting both the input generator and the analysis node.

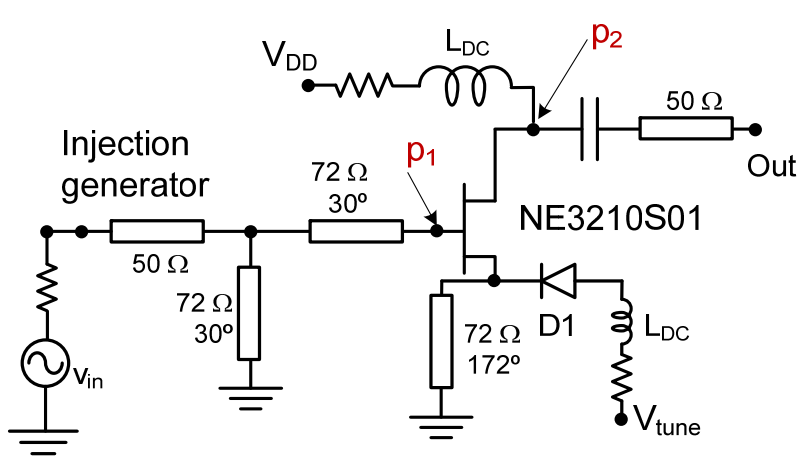

Fig. 1. Schematic of the FET-based injection-locked oscillator. The free-running frequency is $f_{0}=5.185 \mathrm{GHz}$.

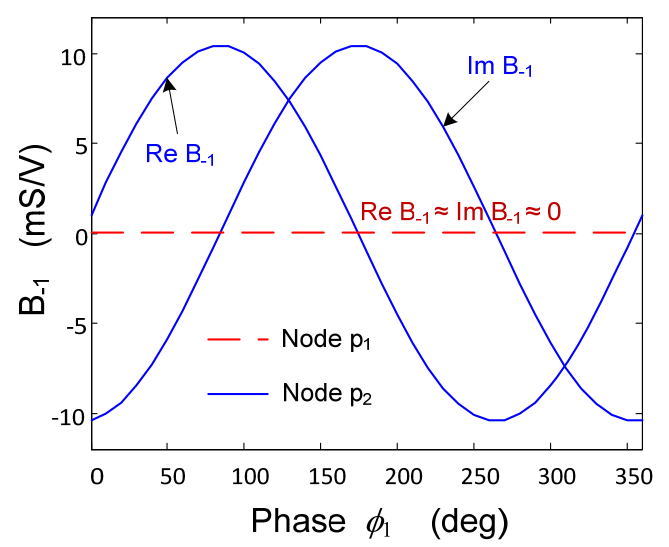

Fig. 2. Phase dependence of the injection sensitivity $B_{-1}\left(\phi_{1}\right)$. For the analysis node $p_{1}$ (transistor gate), the semi-analytical model fulfills $B_{-1}\left(\phi_{1}\right) \simeq 0$ and Cauchy-Riemann conditions (14) hold. On the contrary, for the analysis node $p_{2}$ (transistor drain), the semianalytical model must take into account the sensitivity term $B_{-1}\left(\phi_{1}\right)$.

\section{B. Analysis in the presence of noise sources}

Using the technique in [13], the effect of all the noise sources existing in the oscillator circuit can be modeled with an equivalent current generator connected in parallel at the analysis node. This current generator is a narrow-band signal, which can be expressed as:

$$
i_{n}(t)=2 \operatorname{Re}\left\{I_{n}(t) e^{j \omega t}\right\}, \quad I_{n}(t)=\int_{-B W}^{B W} I_{n}(\Omega) e^{j \Omega t} d \Omega
$$

where $2 B W$ is the noise source frequency bandwidth. On the other hand, the phase noise of the injection source will be modelled as:

$$
U_{m}(t)=U e^{j \phi_{i n}(t)}, \quad \phi_{i n}(t)=\phi_{i n}^{0}+\psi(t)
$$

where $\psi(t)$ is the phase noise process of the input source. The effect of these sources is to perturb the amplitude and phase of the solution harmonics. In order to obtain the equation that determines the dynamics of the perturbed amplitude and phase variables, a similar procedure to the one described in [12], [28] will be carried out. In particular, the first harmonic term of the voltage signal $v_{p}$ at the analysis node becomes:

$$
X_{1}^{p}(t)=\left[V_{1}^{0}+\Delta V(t)\right] e^{j \phi_{1}(t)}
$$


Then, in the presence of the noisy current generator, the admittance equation at node $p$ becomes:

$$
\begin{aligned}
& Y_{p}(t)\left[V_{1}^{0}+\Delta V(t)\right] e^{j \phi_{1}(t)}=I_{n}(t), \\
& Y_{p}(t) \equiv Y_{p}\left[V_{1}^{0}+\Delta V(t), \phi_{1}(t), U_{-m}(t), U_{m}(t), \omega_{0}+\Delta \omega+\frac{s}{j}\right]
\end{aligned}
$$

where $s$ is the complex frequency increment, acting as a time derivative operator. Now, expanding the admittance function $Y_{p}(t)$ as in (6) and neglecting quadratic terms in the perturbation variables we finally obtain:

$$
\begin{aligned}
Y_{V} \Delta V(t)+ & Y_{\omega}\left[\Delta \omega+\dot{\phi}_{1}(t)-j \frac{\Delta \dot{V}(t)}{V_{1}^{0}}\right]+ \\
& +B_{m}^{0} U e^{j\left[\phi_{n}(t)-m \phi_{1}(t)\right]}+B_{-m}^{0} U e^{-j\left[\phi_{m}(t)-m \phi_{1}(t)\right]}=\frac{I_{n}(t)}{V_{1}^{0}}
\end{aligned}
$$

In the following, the time derivative of the amplitude increment $\Delta \dot{V}(t)$ will be neglected in (19) as, due to the amplitude-limiting property of nonlinear elements [12], the magnitude of $\Delta \dot{V}(t) / V_{1}^{0}$ is usually much smaller than that of $\dot{\phi}_{1}(t)$. Equation (19) is the dynamic semi-analytical model of the ILO in the presence of noise sources. In the absence of noise sources, the synchronized oscillator circuit is phase locked to the injection source as predicted by the steady-state model (10). In this state, the phase difference $\varphi=\phi_{i n} / m-\phi_{1}$ remains constant in time, at the value fulfilling equation (12). When noise sources are introduced in the circuit, the phase difference $\varphi(t)$ becomes perturbed. In order to analyze the dynamics of the perturbed phase-locked state, we perform the change of variable $\varphi(t)=\phi_{i n}(t) / m-\phi_{1}(t)$ in equation (19), obtaining:

$$
\dot{\varphi}(t)=\Delta \omega+K_{S} \sin m \varphi+K_{C} \cos m \varphi+\bar{H}^{+} \bar{n}+\frac{\dot{\psi}}{m}
$$

The coefficients of differential equation (20) are:

$$
\begin{aligned}
& K_{S}=-\frac{U\left(B_{m}^{0}-B_{-m}^{0}\right) \cdot Y_{V}}{Y_{\omega} \times Y_{V}}, K_{C}=-\frac{U\left(B_{m}^{0}+B_{-m}^{0}\right) \times Y_{V}}{Y_{\omega} \times Y_{V}} \\
& \bar{H}^{+}=\frac{\left(Y_{V}^{i}-Y_{V}^{r}\right)}{Y_{\omega} \times Y_{V}}, \quad \bar{n}=\frac{1}{V_{1}^{0}}\left(\begin{array}{c}
I_{n}^{r} \\
I_{n}^{i}
\end{array}\right)
\end{aligned}
$$

where the simplifying relation $a \cdot b=a^{r} b^{r}+a^{i} b^{i}$ has been introduced. Note that the coefficients of (20) are determined by the admittance derivatives (6), extracted from circuit-level Harmonic Balance simulations performed on commercial software.

In most cases, it will suffice to consider only Gaussian white noise sources for the following reason. As derived in [13], the oscillator phase noise spectral density $\left\langle\left|\phi_{1}(\Omega)\right|^{2}\right\rangle$ will follow that of the reference source $\left\langle\left|\phi_{\text {in }}(\Omega)\right|^{2}\right\rangle / m^{2}$ up to a certain frequency value $\Omega_{1}$, which is generally above the region of flicker noise influence. As a consequence, the phase difference variable $\varphi=\phi_{i n} / m-\phi_{1}$, which is the relevant one for the present analysis, will not generally be affected by the flicker noise sources. The value of $\left\langle|\varphi(\Omega)|^{2}\right\rangle$ will only become significant for $\Omega>\Omega_{1}$, where the dominant contribution usually comes from the oscillator noise sources. For this reason, the flicker noise sources have not been included in the analysis. For completeness, the component of the source phase noise due to white noise sources will also be included in the analysis, since it may have influence for $\Omega>\Omega_{1}$, especially around $\Omega_{1}$. As derived in [29], this component can be modelled as a Wiener process:

$$
\dot{\psi}(t)=H_{g} n_{g}(t)
$$

where $n_{g}$ is the combined effect of the white noise sources affecting the injection source and $H_{g}$ is a constant term providing the sensitivity to this source. Then, system (20) can be rewritten in terms of the white noise sources as:

$$
\begin{aligned}
& \dot{\varphi}(t)=f\left[\varphi(t), \bar{n}(t), n_{g}(t)\right], \\
& f(\varphi, \bar{n}, \dot{\psi})=\Delta \omega+K_{S} \sin m \varphi+K_{C} \cos m \varphi+\bar{H}^{+} \bar{n}+\frac{H_{g}}{m} n_{g}
\end{aligned}
$$

where the function $f\left(\varphi, \bar{n}, n_{g}\right)$ will be called the restoring term, since it is responsible for recovering the steady-state when the system is perturbed by the noise sources. Equation (23) provides a nonlinear model of the ILO, which will be used hereafter to analyze the random jumps producing cycle slips. The noise sources included in model (23) fulfill:

$$
\begin{aligned}
& \left\langle\bar{n}(t) \bar{n}(t+\tau)^{+}\right\rangle=\left(\begin{array}{ll}
\Gamma & 0 \\
0 & \Gamma
\end{array}\right) \delta(\tau), \\
& \left\langle n_{g}(t) n_{g}(t+\tau)^{+}\right\rangle=\gamma_{g} \delta(\tau)
\end{aligned}
$$

The values $\Gamma$ and $\gamma_{g}$ will be extracted from the phase noise measurement of the free-running oscillator, according to the methodology proposed in [13]. As a result of the introduction of the noise sources in the formulation, the phase variable $\varphi(t)=\phi_{\text {in }}(t) / m-\phi_{1}(t)$ becomes a stochastic process, whose dynamics is ruled by the stochastic differential equation (SDE) (23).

\section{Cycle slipping phenomenon}

In the absence of noise sources, the steady-state solutions of (23) are time-constant synchronized solutions that repeat periodically in the phase variable as:

$$
\varphi_{s k}=\varphi_{s 0}+\frac{2 \pi}{m} k, \quad \varphi_{u k}=\varphi_{u 0}+\frac{2 \pi}{m} k, k \in \mathbb{Z}
$$

with $\varphi_{s 0}, \varphi_{u 0} \in[0,2 \pi / m]$. The solutions $\varphi_{s k}, \varphi_{u k}$ are stable and unstable, respectively. As $\Delta \omega$ approaches any of the two boundaries of the synchronization band, both types of 
solutions get closer, eventually colliding at $\varphi_{s k}=\varphi_{u k}$. The solutions $\varphi_{s 0}, \varphi_{u 0}$ corresponding to the ILO in Fig. 1 have been represented throughout the synchronization band in Fig. 3 , for the case $m=1$.

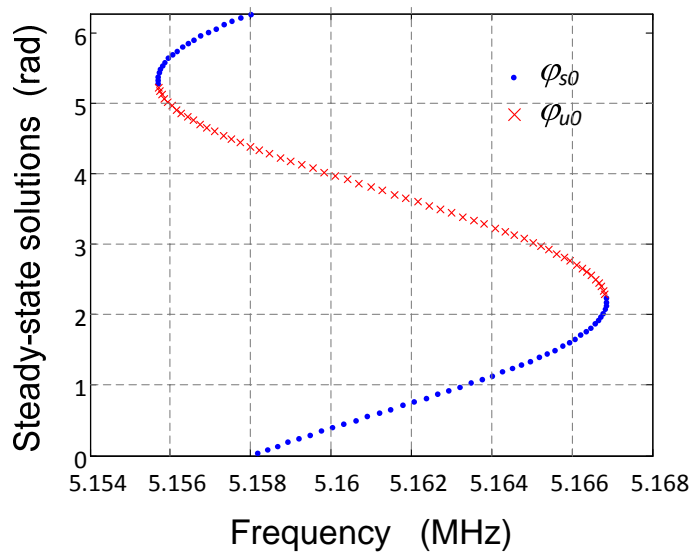

Fig. 3. Steady-state solutions $\varphi_{s 0}, \varphi_{u 0}$ throughout the synchronization frequency interval, for the ILO in Fig. 1 . The case $m=1$ has been selected.

In Fig. 4, several stable and unstable solutions have been represented throughout the phase variable axis. This axis can be divided into intervals $M_{k}$, each one corresponding to the attracting manifold of a stable solution $\varphi_{s k}$. In the absence of noise sources, for any initial condition of system (23) fulfilling $\varphi(0) \in M_{k}$, the restoring term $f(\varphi, \overline{0}, 0)$ pushes the trajectory towards the stable solution $\varphi_{s k}$. The discretization of this trajectory has been schematized in Fig. 4 by the black arrows in each manifold.

When noise sources are introduced into the circuit, the system trajectory fluctuates about a given stable steady-state solution $\varphi_{s k}$. At each time value, if the restoring term satisfies $\operatorname{sign} f\left(\varphi, \bar{n}, n_{g}\right)=\operatorname{sign} f(\varphi, \overline{0}, 0)$, we will say that it is naturally polarized. In this case, the phase step of the discretized trajectory at that time value will agree in sign with the black arrows in Fig. 4. Cycle slips will take place when the noise sources invert the polarity of the restoring term, such that $\operatorname{sign} f\left(\varphi, \bar{n}, n_{g}\right)=-\operatorname{sign} f(\varphi, \overline{0}, 0)$. If the polarity inversion extends over a long enough time interval, the trajectory can be pushed towards the closest unstable solution $\varphi_{u k}$ or $\varphi_{u, k-1}$ throughout the interval $\left(\varphi_{s k}, \varphi_{u k}\right)$ or $\left(\varphi_{u, k-1}, \varphi_{s k}\right)$ (see Fig. 4). If the unstable solution is surpassed, the trajectory enters the stable manifold $M_{k \pm 1}$, associated with $\varphi_{s, k \pm 1}$, evolving to this new solution. During the transient towards $\varphi_{s, k \pm 1}$ the oscillator will be unlocked from the reference for a number of cycles determined by the transient time length. This transition time between two solutions is determined by the stability margin of the stable solution, which can be approximated through perturbation analysis, linearizing (23) about the stable solution $\varphi_{s k}$ :

$$
\begin{aligned}
& \varphi(t)=\varphi_{n k}+\Delta \varphi(t) \\
& \Delta \dot{\varphi}(t)=f\left[\varphi_{n k}+\Delta \varphi(t), \overline{0}, 0\right] \simeq \frac{\partial f\left(\varphi_{n k}, \overline{0}, 0\right)}{\partial \varphi} \Delta \varphi(t)= \\
& =v \Delta \varphi(t) \rightarrow \Delta \varphi(t) \propto e^{v t}, \\
& v=m\left(K_{S} \cos m \varphi_{n k}-K_{C} \sin m \varphi_{n k}\right)
\end{aligned}
$$

The stable pole $v<0$ determines the transient length, which is inversely proportional to the magnitude $|v|$. Then, as the synchronization boundaries are approached, the magnitude $|v|$ decreases, increasing the transition time and the number of reference cycles slipped.

This is a nonlinear phenomenon, which requires the consideration of both the stable and unstable steady state solutions. For this reason, it cannot be predicted by any linear model, obtained from the linearization of ILO equations about the stable solution. Although such a linear model can predict other effects of the noise sources, such as the phase noise characteristic [13], it is unable to predict the cycle slipping phenomenon.

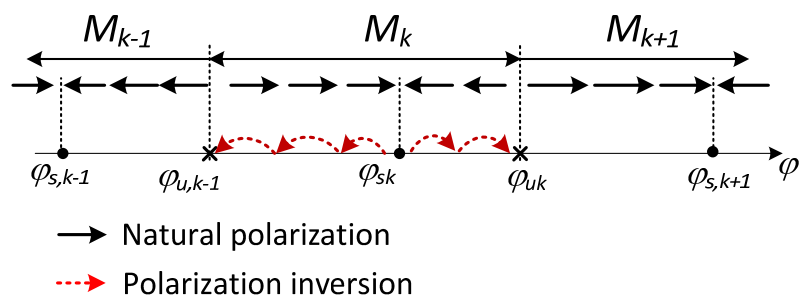

Fig. 4. Schematic of the cycle slipping mechanism. The timediscretized phase trajectory has been represented. Continuous arrows represent the trajectory in absence of noise, while dotted arrows represent the trajectory when noise inverts the restoring term polarity.

The capability of model (23) to predict the cycle slipping is shown in Fig. 5, where a time realization of the simulated phase process $\varphi(t)$ is represented. In this simulation, the model coefficients (6) have been extracted from the ILO in Fig. 1 . The case $m=1$ has been selected. For comparison, the envelope transient simulation of this circuit in commercial Harmonic Balance has been superimposed. Both techniques predict a similar pattern of cycle slips in the time interval considered. The jumps take place at random times. The differences in the jump times between both analyses can be explained in the following way: on the one hand, the envelope-transient model used in commercial software works with a system of $q(2 N H+1)$ state variables, where $q$ is the number of independent variables and $\mathrm{NH}$ is the number of harmonic components used for each variable. On the other hand, the semi-analytical model (23) contains only one state variable. In [22] the transient dynamical behavior of the semianalytical model in absence of noise has been analyzed, verifying that it agrees with the envelope-transient results in commercial software for any time realization. This is because the transient dynamics in absence of noise is structurally stable [30], that is, the inaccuracies associated with the model reduction produce small changes in the time paths. In the 
analysis of the random jumps, the situation is different. A phase jump in a single time realization is produced by specific sequences of consecutive values of the noise sources that alter the state variables in a particular way [1]. This dynamics is not structurally stable, since under any slight change in the model a given jump-producing sequence may stop generating a jump. As a consequence, a sequence of values in a noise realization producing a phase jump in the envelope-transient model may not affect in the same way the semi-analytical model. In spite of this mismatch, the statistical results of both models will approximately agree, since the white noise sources cover all the possible combination of sequences of values.

As can be seen in the figure, during each jump the oscillator loses phase lock and slips about one thousand reference periods.

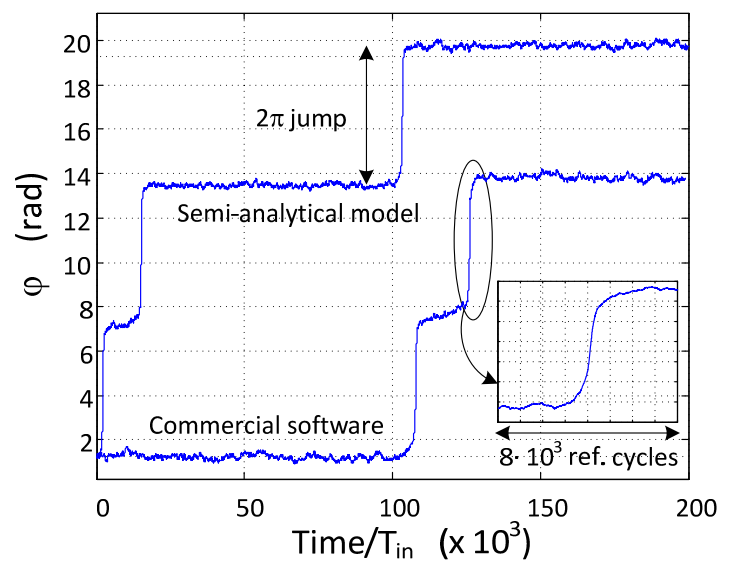

Fig. 5. Time realization of the phase process. Cycle slipping phenomenon for the ILO in Fig. 1, with $f_{i n}=\omega_{i n} /(2 \pi)=1 / T_{i n}=5.18 \mathrm{GHz}$.

The objective of this work is to obtain the stochastic properties of the phase shift variable $\varphi(t)$ in the presence of noise sources. The knowledge of these properties will enable the determination of the influence of the ILO parameters on the stochastic behavior of the system, in particular the cycle slipping phenomenon. Note that the computational cost of the commercial software simulation in Fig. 5 is very high. This is because, in this case, the required time step for the envelope transient to converge was $\Delta t \approx T_{\text {in }}$, with $T_{\text {in }}=2 \pi / \omega_{\text {in }}$, whereas the time length is $2 \cdot 10^{5} T_{\text {in }}$. The envelope simulation in Fig. 5 in commercial software took about 2 hours (Intel Xeon X5450@3 GHz / 16 GB RAM), whereas the simulation of the semi-analytical model took about 0.6 seconds. The integration time step of the semi-analytical model has been set to $\Delta t=1 /(4|v|)$. Note that the envelope formulation makes use of the models of the distributed elements in the circuit. As a consequence, a Monte-Carlo type analysis of $\varphi(t)$ based on envelope transient simulation would require a very high computational cost, rendering it inefficient. In the next Section, an alternative technique will be proposed for the stochastic analysis of the ILO.

\section{StOChastiC ANALYSIS OF THE INJECTED OSCILLATOR}

In this section, the stochastic properties of the phase process $\varphi(t)$ will be analyzed with the Fokker-Planck equation [1][24] associated with the stochastic differential equation (23):

$$
\frac{\partial p(\varphi, t)}{\partial t}=\left[-\frac{\partial}{\partial \varphi} k_{1}(\varphi)+\frac{k_{2}}{2} \frac{\partial^{2}}{\partial \varphi^{2}}\right] p(\varphi, t) \equiv L p(\varphi, t)
$$

where $p(\varphi, t)$ is the time-varying probability density function (PDF) of the phase process and $L$ is the so-called FokkerPlanck operator. The terms $k_{1}$ and $k_{2}$ are extracted from the SDE (20) [24]:

$$
\begin{aligned}
& k_{1}(\varphi)=K_{S} \sin m \varphi+K_{S} \cos m \varphi+\Delta \omega, \\
& k_{2}=|\bar{H}|^{2} \Gamma+\frac{H_{g}^{2}}{m^{2}} \gamma_{g}
\end{aligned}
$$

Equation (27) is a partial differential equation that can be efficiently solved due to the reduced number of independent variables $(\varphi, t)$. In order to solve equation (27) it is necessary to fix a set of boundary conditions on the PDF function $p(\varphi, t)$. The method of resolution will depend on the kind of boundary conditions imposed. In the following, two different kinds of boundary conditions will be proposed, applying the resulting PDFs to the analysis of the cycle slipping probability.

\section{A. Periodic boundary conditions}

In this approach, the probability space is reduced to the interval $\varphi \in S_{2} \equiv[0,4 \pi / \mathrm{m}]$, by mapping the realizations of the stochastic process $\varphi(t)$ to the space $S_{2}$ through the modulo $4 \pi / m$ function. As a consequence, the PDF of the process $\varphi(t)$ fulfills:

$$
\begin{aligned}
& p(\varphi=0, t)=p(\varphi=4 \pi / m, t), \\
& p(\varphi, t)=0, \quad \varphi \notin[0,4 \pi / m]
\end{aligned}
$$

In this probability space, the stable solutions $\varphi_{s, 2 k}$ and $\varphi_{s, 2 k+1}$ are respectively mapped to the solutions $\varphi_{s 0}, \varphi_{s 1} \in S_{2}$. Then, the function $p(\varphi, t)$ determines the transitions between the two attractors $\varphi_{s 0}$ and $\varphi_{s 1}$. Considering the boundary conditions (29), the PDF $p(\varphi, t)$ can be calculated by solving (27) for a phase-periodic function $q(\varphi, t)$ fulfilling:

$$
\begin{aligned}
& q(\varphi, t)=q(\varphi+4 \pi / m, t), \quad \int_{0}^{4 \pi / m} q(\varphi, t) d \theta=1, \quad \forall t \\
& p(\varphi, t)=q(\varphi, t), \quad \varphi \in[0,4 \pi / m]
\end{aligned}
$$

As can be seen in equation (30), the $\operatorname{PDF} p(\varphi, t)$ agrees with the periodic function $q(\varphi, t)$ within the interval $S_{2}$. In order to calculate $q(\varphi, t)$, an expansion of this function in a basis of linearly independent functions will be considered, as proposed in [1],[24]: 


$$
q(\varphi, t)=\sum_{k} b_{k} q_{k}(\varphi, t)
$$

where $b_{k}$ are coefficients determined by the initial condition $q(\varphi, 0)$. In order for $q(\varphi, t)$ to fulfill (27) and conditions (30), the following properties are required for the functions $q_{k}(\varphi, t)$

$$
\begin{aligned}
& \frac{\partial q_{k}(\varphi, t)}{\partial t}=L q_{k}(\varphi, t) \\
& q_{k}(\varphi, t)=q_{k}(\varphi+4 \pi / m, t)
\end{aligned}
$$

To solve the functions $q_{k}(\varphi, t)$, the method of separation of variables [31] will be applied, using the decomposition:

$$
q_{k}(\varphi, t)=h_{k}(t) q_{k}(\varphi)
$$

Applying condition (32)(a) to expression (33) we obtain

$$
\frac{\dot{h}_{k}(t)}{h_{k}(t)}=\frac{L q_{k}(\varphi)}{q_{k}(\varphi)}=-\lambda_{k}
$$

where $-\lambda_{k}$ is the required separation constant [31]. Expression (34) provides the relations:

$$
h_{k}(t)=e^{-\lambda_{k} t}, \quad L q_{k}(\varphi)=-\lambda_{k} q_{k}(\varphi)
$$

where the second equation in (35) indicates that $\lambda_{k}$ and $q_{k}(\varphi)$ are respectively the eigenvalues and the eigenfunctions of the operator $L$. Since, by condition (32)(b) the eigenfunctions $q_{k}(\varphi)$ are periodic, they can be expanded in a Fourier series as:

$$
q_{k}(\varphi)=\sum_{l=-M}^{M} c_{l k} \Psi_{l}(\varphi), \quad \Psi_{l}(\varphi) \equiv \exp \left(j l \frac{m \varphi}{2}\right)
$$

where $M$ is the number of harmonics considered. Introducing expansion (36) into equation (35), the following algebraic system is obtained:

$$
A \bar{c}_{k}=\lambda_{k} \bar{c}_{k}, \quad A \equiv\left(A_{r l}\right)
$$

where each vector $\bar{c}_{k}$ contains the $2 M+1$ coefficients $c_{l k}$ of the Fourier expansion (36). The matrix $A$ contains $(2 M+1 \times 2 M+1)$ components given by the coefficients of the endomorphism $L$ in the basis $\left\{\Psi_{l}(\varphi)\right\}_{l=-M}^{M}$ :

$$
\begin{aligned}
& L \Psi_{l}(\varphi)=\sum_{r=-M}^{M} A_{r l} \Psi_{r}(\varphi), \\
& A_{l-2, l}=K_{T}^{*}(l / 4-1 / 2), \quad A_{l+2, l}=-K_{T}(l / 4+1 / 2), \\
& A_{l, l}=\left(-j 4 m l \Delta \omega+m^{2} l^{2} k_{2}\right) / 8, \quad K_{T}=m\left(K_{S}+j K_{C}\right)
\end{aligned}
$$

where only the components $A_{1 \pm 2, l}, A_{l, l}$ are non-zero. From (37) , once the components of the matrix $A$ are obtained, the stochastic analysis reduces to the simple calculation of the eigenvalues and eigenvectors of the matrix A. Finally, the PDF $p(\varphi, t)$ associated with system (20) is expressed as:

$$
p(\varphi, t)=\sum_{k=1}^{2 M+1} b_{k} e^{-\lambda_{k} t} \sum_{l=-M}^{M} c_{l k} \exp \left(j l \frac{m \varphi}{2}\right), \quad \varphi \in[0,4 \pi / m]
$$

The coefficients $b_{k}$ depend on the initial condition $p(\varphi, 0)$, which can be expressed in the periodic Fourier basis as:

$$
p(\varphi, 0)=\sum_{l=-M}^{M} u_{l} \Psi_{l}(\varphi)
$$

On the other hand, expansion (39) for $t=0$ provides:

$$
p(\varphi, 0)=\sum_{k=1}^{2 M+1} b_{k} \sum_{l=-M}^{M} c_{l k} \Psi_{l}(\varphi)
$$

Then, equating expressions (40) and (41), and considering that $\left\{\Psi_{l}(\varphi)\right\}_{l=-M}^{M}$ is a complete basis, we arrive at:

$$
\bar{b}=C^{-1} \bar{u}, \quad C=\left[\begin{array}{lll}
\bar{c}_{1} & \cdots & \bar{c}_{2 M+1}
\end{array}\right]
$$

where $\bar{b}$ is the vector containing the $2 M+1$ coefficients $b_{k}$ and $\bar{u}$ contains the $2 M+1$ harmonic components of expansion (40). Equation (39) enables the time evolution of the stochastic properties of process $\varphi(t)$ to be obtained. It can be applied to several analyses, such as the study of the influence of the noise sources on the locking transient length. To summarize, the technique to obtain analytical expression (39) for the PDF $p(\varphi, t)$ is based on four steps:

1- Calculate the coefficients of the semi-analytical model (20) through commercial Harmonic Balance simulation of the ILO circuit. This simulation is carried out in the absence of noise sources.

2- Obtain matrix $A$ using equations (38) by means of the coefficients calculated in Step 1.

3- Calculate the set of eigenvalues $\lambda_{k}$ and eigenvectors $\bar{C}_{k}$ of matrix $A$.

4- Calculate the coefficients $b_{k}$ utilizing equation (42), by making use of the harmonic components of the initial condition function $p(\varphi, 0)$.

Here, expression (39) will be used to analyze the cycle slipping phenomenon. For this purpose, a Gaussian-type initial distribution $p(\varphi, 0)$ centered at $\varphi_{s 0}$ has been chosen. The analysis has been applied to the ILO in Fig. 1. After obtaining expression (39) by following Steps 1-4, the time evolution of the PDF $p(\varphi, t)$ has been represented in Fig. 6. As time evolves, there is a probability flow between $\varphi_{s 0}$ and $\varphi_{s 1}=\varphi_{s 0}+2 \pi / m$ due to random jumps, until the steady state distribution $p(\varphi, t \rightarrow \infty)$ is reached. In this state, all the functions $q_{k}(\varphi, t)$ in expansion (31) vanish except $q_{1}(\varphi, t)$, associated with the eigenvalue $\lambda_{1}=0$. Then, the steady state is given by:

$$
p(\varphi, \infty) \equiv p(\varphi, t \rightarrow \infty)=b_{1} q_{1}(\varphi)
$$




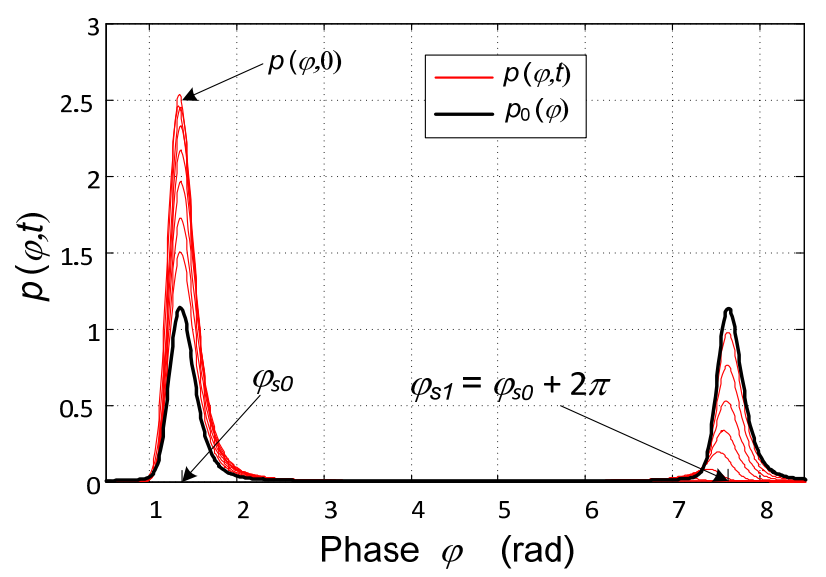

Fig. 6. Time evolution of the probability density function for $\mathrm{f}_{\mathrm{in}}=5.18$ $\mathrm{GHz}$, with $\Gamma=10^{-18} \mathrm{~S}^{2}, H_{g}^{2} \gamma_{g}=39.48 \mathrm{~Hz}^{-2}$. The case $m=1$ has been selected.

Let us arrange the set of eigenvalues as:

$$
0=\lambda_{1}<\lambda_{2}^{r}<\cdots<\lambda_{2 M+1}^{r}
$$

where the super-index $r$ means real part. The probability of jumps is inversely proportional to the time $t_{s}$ needed to reach the steady state $p(\varphi, \infty)$. By inspection of expansion (39), it is seen that $t_{s}$ decreases with $\lambda_{k}^{r}$, making the probability of jumps proportional to $\lambda_{2}^{r}$. To analyze this probability, expansion (39) will be approximated by:

$$
p(\varphi, t) \simeq b_{1} q_{1}(\varphi)+b_{2} e^{-\lambda_{2} t} q_{2}(\varphi), \quad \varphi \in[0,4 \pi]
$$

It must be noted that approach (45) is based on the assumption that $\lambda_{2} \in \mathbb{R}$ and $\lambda_{2} \ll \lambda_{3}^{r}$. In the cases where this condition is not fulfilled, the following analysis is not valid and the method in Section III.B must be applied. The probability space can be divided into two non-intersecting regions I and II, each one containing one of the stable solutions $\varphi_{s 0}, \varphi_{s 1}$, fulfilling:

$$
\int_{I} p(\varphi, \infty) d \varphi=\int_{I I} p(\varphi, \infty) d \varphi=\frac{1}{2}
$$

Applying approach (45), the time-varying probability of each region is given by:

$$
\begin{aligned}
& \begin{aligned}
p_{I}(t) & =\int_{I} p(\varphi, t) d \varphi \simeq \int_{I}\left[b_{1} q_{1}(\varphi)+b_{2} e^{-\lambda_{2} t} q_{2}(\varphi)\right] d \varphi= \\
& =A_{I}+B_{I} e^{-\lambda_{2} t},
\end{aligned} \\
& p_{I I}(t)=\int_{I I} p(\varphi, t) d \varphi=A_{I I}+B_{I I} e^{-\lambda_{2} t}, \\
& p_{I}(t)+p_{I I}(t)=1, \quad \forall t
\end{aligned}
$$

Starting from the initial condition $\varphi(0)=\varphi_{s 0}$, we define $T_{s}$ as the time at which the first jump is produced. Once the adjacent attractor $\varphi_{s 1}$ is reached, the time until the next jump can be simulated in system (20) by resetting the time variable to $t=0$ and using the initial condition $\varphi(0)=\varphi_{s 0}$ again. Then,
$T_{s}$ is a stochastic variable that provides the time between two consecutive jumps. This variable can be analyzed by means of system (47). At time $t=0$ all the probability is concentrated at the stable solution $\varphi_{s 0}$, providing:

$$
p(\varphi, 0)=\delta\left(\varphi-\varphi_{s 0}\right) \rightarrow p_{I}(0)=1, p_{I I}(0)=0
$$

Combining expressions (46)-(48), we arrive at:

$$
\begin{aligned}
& \dot{p}_{I}(t)=-J_{12}(t)+J_{21}(t), \\
& \dot{p}_{I I}(t)=J_{12}(t)-J_{21}(t), \\
& J_{12}(t)=\frac{\lambda_{2}}{2} p_{I}(t), \quad J_{21}(t)=\frac{\lambda_{2}}{2} p_{I I}(t)
\end{aligned}
$$

where $J_{i j}(t)$ provides the time rate of the probability of leaving region $i$ towards region $j$ at time $t$. Then, the probability of a jump occurring at a given time $t$ is:

$$
\begin{aligned}
& P\left(t<T_{s}<t+d t\right)=\frac{J_{12}(t) d t}{\int_{0}^{\infty} J_{12}(t) d t}=\frac{\lambda_{2}}{2} \exp \left(\frac{\lambda_{2}}{2} t\right) d t= \\
& =\chi(t) d t
\end{aligned}
$$

where $\chi(t)$ is the PDF of the stochastic variable $T_{s}$. Expression (50) can be applied to calculate the mean value of the time between two consecutive jumps:

$$
E\left[T_{s}\right]=\int_{0}^{\infty} t \chi(t) d t=\frac{2}{\lambda_{2}}
$$

This result will be applied in Section III.C to analyze the influence of the ILO parameters on the mean time between jumps.

\section{B. Absorbing boundaries}

In this approach, the probability space is reduced to the interval $\varphi \in\left[\varphi_{s,-1}, \varphi_{s, 1}\right]=\left[\varphi_{s 0}-2 \pi / m, \varphi_{s 0}+2 \pi / m\right]$. It is assumed that when a realization of the phase process reaches one of the boundaries $\varphi_{s,-1}, \varphi_{s, 1}$ it is absorbed and therefore eliminated from the ensemble. The fluctuating nature of the realizations increases the probability of being absorbed as the boundaries are approached. In the limit, the PDF of the phase process $\varphi(t)$ fulfills the boundary conditions:

$$
p\left(\varphi_{s 0}-2 \pi / m, t\right)=p\left(\varphi_{s 0}+2 \pi / m, t\right)=0
$$

In this approach, the function $p(\varphi, t)$ provides the amount of probability remaining in the interval $\left[\varphi_{s 0}-2 \pi / m, \varphi_{s 0}+2 \pi / m\right]$ at a given time $t$. The function $p(\varphi, t)$ reduces with time due to the random jumps, until all the probability abandons the interval. This behavior provides the limit $p(\varphi, t \rightarrow \infty)=0$.

In order to calculate $p(\varphi, t)$, it will be expanded in a basis of linearly independent functions:

$$
p(\varphi, t)=\sum_{k} b_{k} p_{k}(\varphi) e^{-\eta_{k} t}
$$


where $b_{k}$ are coefficients determined by the initial condition $p(\varphi, 0)$. Inserting (52) in the Fokker Planck equation (27) we obtain the following system:

$$
\begin{aligned}
& L p_{k}(\varphi)=-\eta_{k} p_{k}(\varphi)=0 \rightarrow \\
& \rightarrow \frac{k_{2}}{2} \frac{d^{2} p_{k}(\varphi)}{d \varphi^{2}}-\frac{d}{d \varphi}\left[k_{1}(\varphi) p_{k}(\varphi)\right]+\eta_{k} p_{k}(\varphi)=0, \\
& p_{k}\left(\varphi_{s 0}-2 \pi / m\right)=p_{k}\left(\varphi_{s 0}+2 \pi / m\right)=0
\end{aligned}
$$

System (54) can be transformed into a Sturm-Liouville twopoint boundary value problem by means of the change of variable [1]:

$$
u_{k}(\varphi)=a(\varphi) p_{k}(\varphi), a(\varphi)=\exp \left\{-\frac{1}{k_{2}} \int_{\varphi_{s k}}^{\varphi} k_{1}(\varphi) d \varphi\right\}
$$

By transformation (55), equation (54) becomes:

$$
\begin{aligned}
& \frac{d^{2} u_{k}(\varphi)}{d \varphi^{2}}+I(\varphi) u_{k}(\varphi)=0, \\
& u_{k}\left(\varphi_{s 0}-2 \pi / m\right)=u_{k}\left(\varphi_{s 0}+2 \pi / m\right)=0, \\
& I(\varphi)=-\frac{1}{k_{2}} \frac{d k_{1}(\varphi)}{d \varphi}-\left[\frac{k_{1}(\varphi)}{k_{2}}\right]^{2}+\frac{2 \eta_{k}}{k_{2}}
\end{aligned}
$$

System (56) is a Sturm-Liouville in the self-adjoint form, providing a discrete set of solutions $\left\{u_{k}(\varphi), \eta_{k}\right\}$ [31]. The set of eigenvalues can be arranged as [31]:

$$
0<\eta_{1}<\eta_{2}<\cdots<\eta_{k}<\cdots<\infty
$$

where $\eta_{k}$ are real numbers. The analysis performed with periodic boundary conditions is usually less demanding than the one using absorbing boundaries. This is because the former reduces to a simple eigenvalue problem, approaching the jumping rate by the eigenvalue $\lambda_{2}$. When using absorbing boundaries, the values $\eta_{k}$ must be calculated through numerical resolution of equation (56) by shooting, finite differences or other numerical techniques [32], which can be more involved than the method of periodic boundaries.

As demonstrated in the involved derivations of seminal work [1], when extracting the coefficients of Sturm-Liouville system (54) from a SDE with the structure (23), the first eigenvalue $\eta_{1}$ is in general several orders of magnitude smaller than the rest. Then, the probability in the interval $\left[\varphi_{s 0}-2 \pi / m, \varphi_{s 0}+2 \pi / m\right]$ at a given time $t$ can be approximated as:

$$
\begin{array}{rl}
p(t)=\int_{\varphi_{s_{0}}-2 \pi / m}^{\varphi_{s_{0}}+2 \pi / m} & p(\varphi, t) d t \simeq \\
& \simeq e^{-\eta_{1} t} \int_{\varphi_{s_{0}}-2 \pi / m}^{\varphi_{s_{0}}+2 \pi / m} p_{1}(\varphi) d t=P_{1} e^{-\eta_{1} t}
\end{array}
$$

Note that, in contrast to the previous method using periodic boundaries, equation (58) can be used in a wider range of specifications, since $\eta_{1} \ll \eta_{2}$ is generally fulfilled. Using (58), the probability of a jump occurring at a given time $t$ is:

$$
P\left(t<T_{s}<t+d t\right)=\frac{\dot{p}(t) d t}{\int_{0}^{\infty} \dot{p}(t) d t}=\eta_{1} e^{-\eta_{1} t} d t=\rho(t) d t
$$

where $\rho(t)$ is the PDF of the stochastic variable $T_{s}$. Expression (59) can be applied to calculate the mean value of the time between two consecutive jumps:

$$
E\left[T_{s}\right]=\int_{0}^{\infty} t \rho(t) d t=\frac{1}{\eta_{1}}
$$

If the approach in Section III.A holds, the mean time between jumps provided by expressions (51) and (60) must agree. The results of the analyses in Sections III.A-B will be compared in Section III.C.

\section{Influence of the ILO parameters on the cycle slipping phenomenon}

In Fig. 7, a schematic of the restoring term $f(\varphi, \overline{0}, 0)$ in equation (23) for $\Delta \omega<0$ is shown. As was indicated in Section II, the random jumps are produced when the noise component in (23) inverts the polarity of the restoring term in the interval $\left[\varphi_{u k}, \varphi_{s k}\right]$. The maximum value of $f(\varphi, \overline{0}, 0)$ in that interval is marked in the figure as $f_{M}(\Delta \omega)>0$. By performing a similar analysis for $\Delta \omega>0$, a value $f_{m}(\Delta \omega)=-f_{M}(-\Delta \omega)<0$ is obtained. As can be seen in the figure, if $\Delta \omega$ is decreased towards the limit of the band, both the stable and unstable solutions get closer and the maximum value $f_{M}$ is decreased. As a consequence, the probability that the noise component inverts the polarity of the restoring term increases.

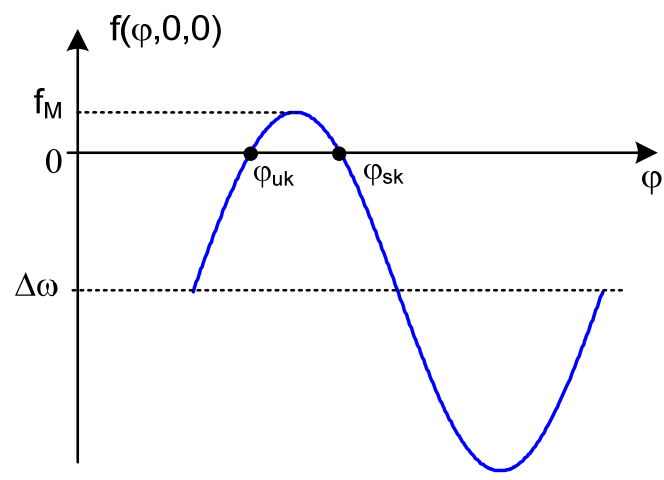

Fig. 7. The restoring term versus the phase difference variable. A phase jump will take place when the polarity of $f(\varphi, \overline{0}, 0)$ is inverted in the interval $\left[\varphi_{u k}, \varphi_{s k}\right]$. In that case, the noise component must exceed the boundary $f_{M}(\Delta \omega)$.

In practice, the amplitude of the noise component is bounded. Here, the following approach will be applied:

$$
\left|\bar{H}^{+} \bar{n}(t)+\frac{H_{g}}{m} n_{g}(t)\right| \leq \sigma_{w}, \forall t, \quad \sigma_{w}^{2}=|\bar{H}|^{2} \Gamma \Omega_{B}+\frac{H_{g}^{2}}{m^{2}} \gamma_{g} \Omega_{B}(61)
$$


where $\sigma_{w}^{2}$ is the variance of the white noise term and $\Omega_{B}$ is the noise bandwidth. Since the noise component is bounded by relation (61), the jumps are possible up to a given frequency distance from the border of the synchronization band. The region of the frequency shift interval where jumps are possible will be called the jump region. This region is composed by two intervals $\left[-\Delta \omega_{\max }, \Delta \omega_{l}\right]$ and $\left[\Delta \omega_{r}, \Delta \omega_{\max }\right]$, where $\mp \Delta \omega_{\max }=\mp 2 \pi \Delta f_{\max }$ are the left and right limits of the synchronization band calculated by means of equation (13). The limits of the jump region can be derived from (61) as:

$$
f_{M}\left(\Delta \omega_{l}\right)=-f_{m}\left(\Delta \omega_{r}\right)=\sigma_{w}
$$

In order to show the capabilities of the simulation techniques in Sections II.A-B, the probability of random jumps has been analyzed throughout the synchronization interval. For this analysis, the fundamentally-synchronized BJT-based oscillator in Fig. 8 with free-running frequency $f_{0}=1 \mathrm{GHz}$ has been chosen. The results have been validated through three different simulations. According to expressions (51) and (60), the jump rate or the mean value of the number of jumps per unit of time is given by $N_{t}=\lambda_{2} / 2$ or $N_{t}=\eta_{1}$, depending on the analysis applied. In Fig. 9(a), the jump rates predicted by the approaches in Sections II.A-B have been shown. As predicted by the qualitative analysis of Fig. 7, the jump rate becomes reduced when entering the center of the synchronization band, where the ILO is more protected against random jumps. In order to consider the cycle slipping phenomenon throughout the whole synchronization band, the power spectral density of the noise sources has been chosen such that the jump region covers the whole range $\left[-\Delta \omega_{\max }, \Delta \omega_{\max }\right]$. In the same figure, the jump rate obtained through Monte-Carlo analysis of system (20) has been superimposed.

In Fig. 9(b) the simulation time of the three methods has been represented versus the injection frequency offset. For a better comparison, the logarithm of the simulation time has been represented. The CPU time for the Fokker-Planck methods remains steady along the synchronization band. As can be seen, the method using periodic boundaries is in general faster than the rest. For the analysis using absorbing boundaries a method based on the shooting technique has been used. The Monte Carlo analysis of the semi-analytical model is faster that the method of absorbing boundaries near the border of the synchronization band. Nevertheless, the simulation time of the Monte Carlo analysis grows exponentially as the injection frequency is moved towards the center of the band, and the method of absorbing boundaries becomes more efficient. For that reason, only Monte Carlo simulations up to $50 \%$ of the synchronization band have been performed.

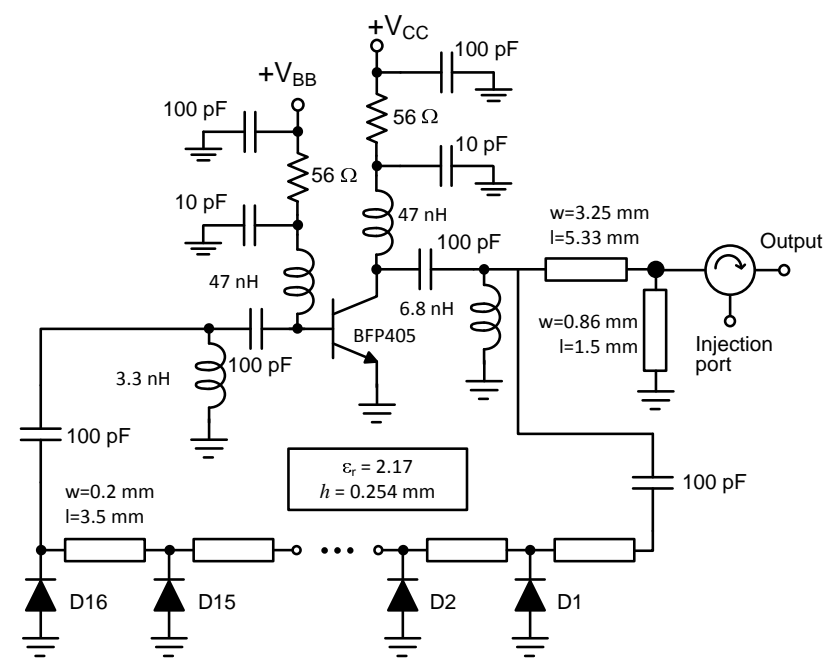

(a)

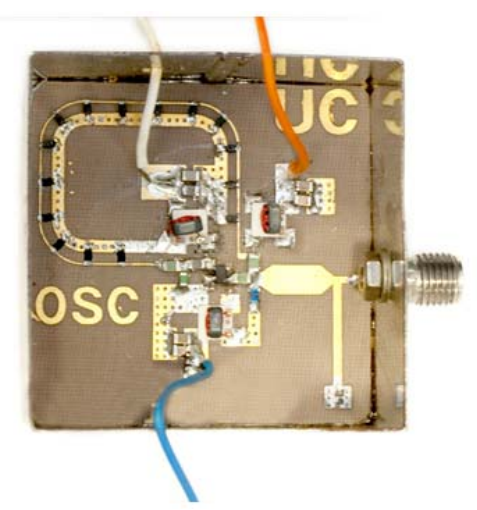

(b)

Fig. 8. BJT-based injection-locked oscillator. The free-running frequency is $f_{0}=1 \mathrm{GHz}$ (a) Schematic. (b) Picture.

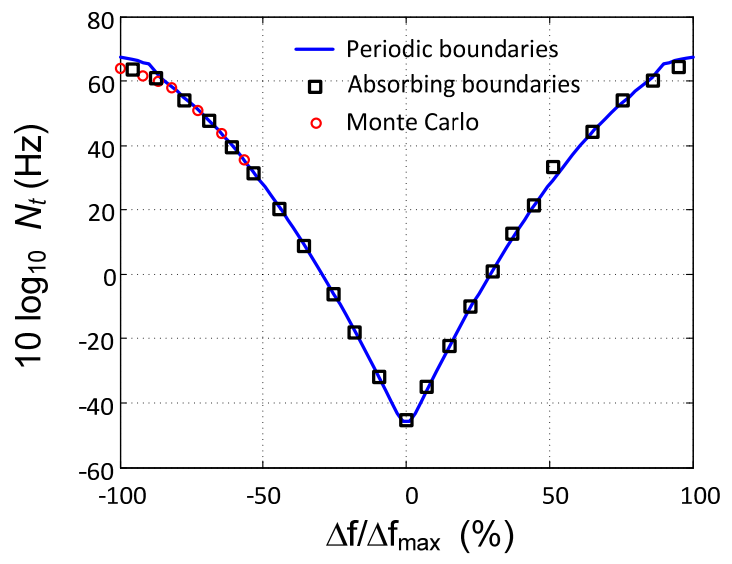

(a) 


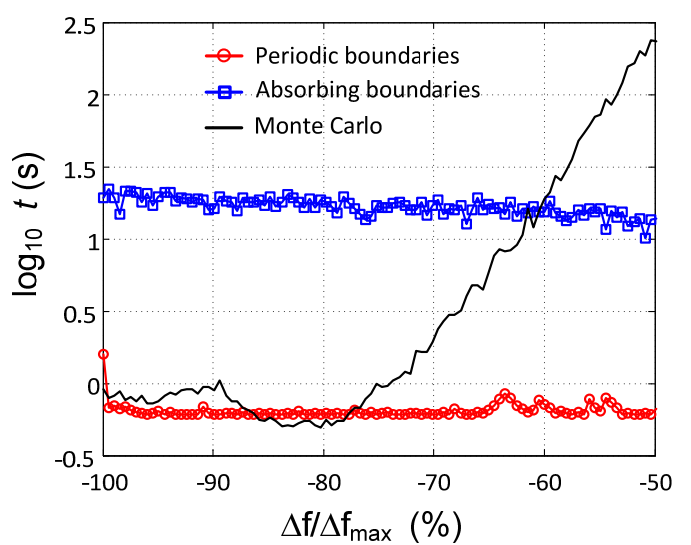

(b)

Fig. 9. (a) Evolution of the jump rate throughout the synchronization band, for $\Gamma=10^{-14} \mathrm{~S}^{2}, H_{g}^{2} \gamma_{g}=39.48 \mathrm{~Hz}^{-2}$. The probability of jump increases as $\Delta f=\Delta \omega / 2 \pi$ approaches the borders of the synchronization band. (b) Comparison of the CPU time required to obtain the three sets of results.

\section{EXPERIMENTAL RESULTS}

\section{A. Time domain measurements}

Fig. 10 shows the measurement setup used for experimental validation. The phase shift between the two signal generators, SG1 and SG2, which are connected to the same $10 \mathrm{MHz}$ reference clock, can be adjusted in order to improve the output response of the phase detector. The output of the phase detector is connected to a digital oscilloscope (Agilent Infiniium DSO90804A) after passing through a low-pass filter. A directional coupler is also connected to the output of the oscillator in order to allow the connection a spectrum analyzer. The spectrum analyzer is used to verify injectionlocked operation and to monitor the output of the ILO to adjust the output power of SG2 at each input frequency value. The output of SG1 is used to synchronize / injection-lock the oscillator circuit. Note that the frequency of the second generator is set equal to the frequency of SG1, while its amplitude is adjusted to match the output of the oscillator. The two inputs of the phase detector (PD) correspond to signals with phase shift $\Delta \phi(t)$ and same frequency and amplitude. If no phase jumps are produced the output of the phase detector is constant. In case a phase jump takes place, the variation of $\Delta \phi(t)$ triggers the oscilloscope.

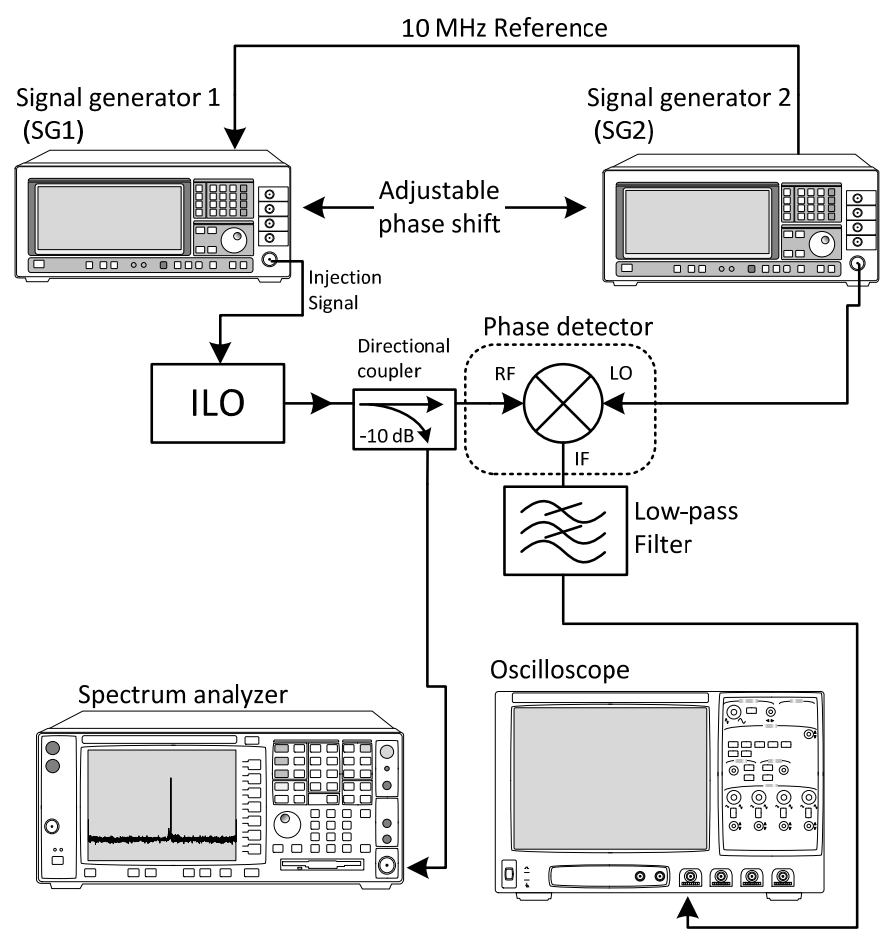

(a)

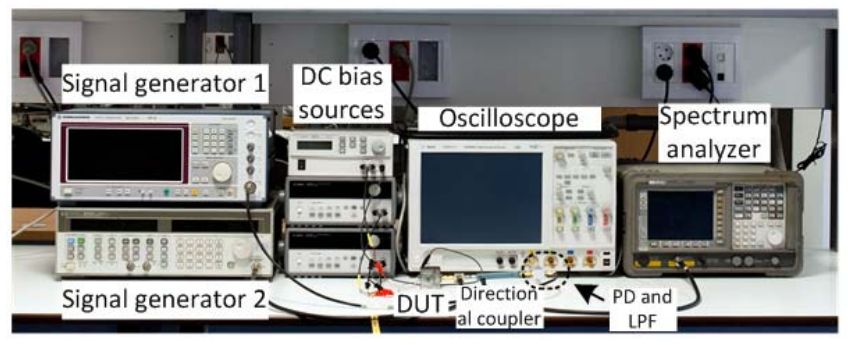

(b)

Fig. 10. Measurement setup used for experimental validation. (a) Schematic. (b) Picture.

The measurements have been carried out in the fundamentally-synchronized BJT-based oscillator in Fig. 8. In Fig. 11, several time-domain measurements of the PD output have been shown. As can be seen in Figs. 11(a,b), as the frequency shift approaches the border or the synchronization band the number of phase jumps increases, in agreement with the previous theoretical results. Note that, as a difference from the case when the ILO operates out of the synchronization band, the jumps do not repeat periodically. This can be observed in Fig. 11(c), where a zoom of Fig. 11(b) has been performed to show two consecutive jumps close in time.

In Fig. 12 the mean time between jumps $E\left[T_{s}\right]$ has been measured throughout the synchronization band. For this purpose, a group of $N=10$ measurements, corresponding to a time interval of $50 \mathrm{~ms}$ have been obtained. As can be seen in the figure, the value $E\left[T_{s}\right]$ increases as the frequency shift is reduced. This is in agreement with both the theoretical and simulation results of the stochastic simulation technique, which have been superimposed in the figure. The higher value 
of the measured $E\left[T_{s}\right]$ near the synchronization border is attributed to the fact that some phase jumps might not be detected by the measurement technique, increasing the expected value of the measured time between jumps. In addition, the discrepancies occur near the borders of the synchronization band. These borders correspond to saddlenode bifurcation points of the ILO system, where the phase difference between the stable and unstable synchronized solutions is very sensitive to small changes in the system parameters. This is due to the infinite slope of the phase shift with respect to the injection frequency (Fig. 3). As a consequence, in this region the probability of jumps predicted by the semi-analytical model can exhibit additional discrepancies with respect to the measurement.

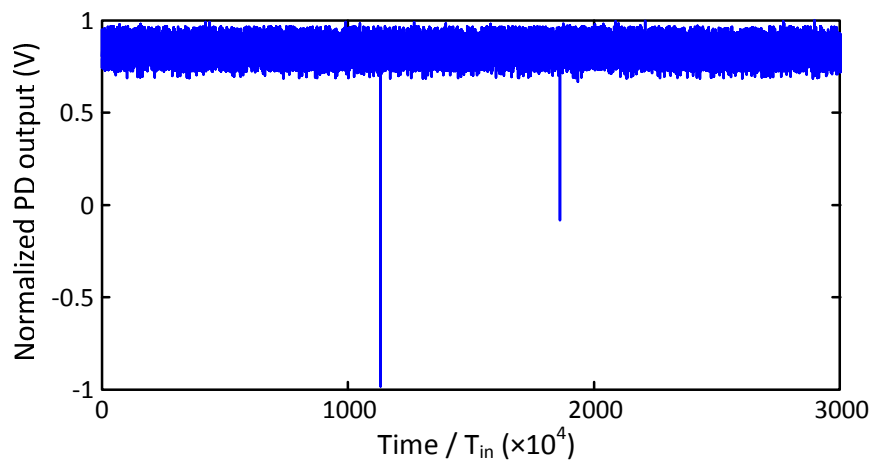

(a)

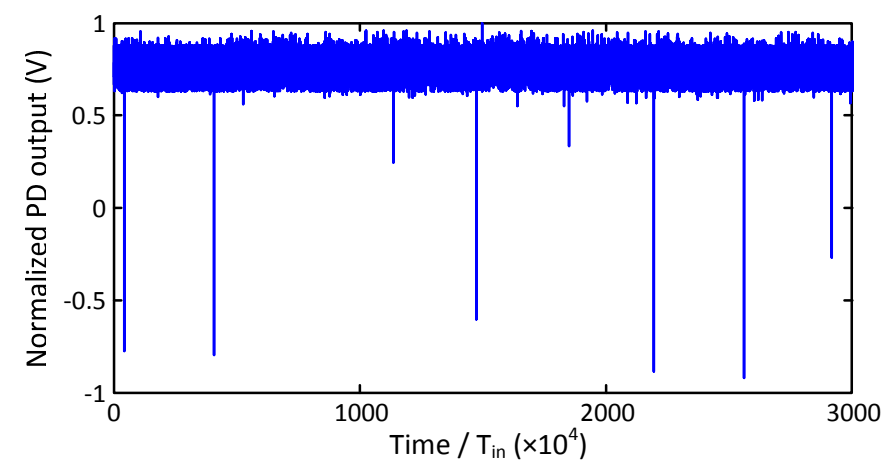

(b)

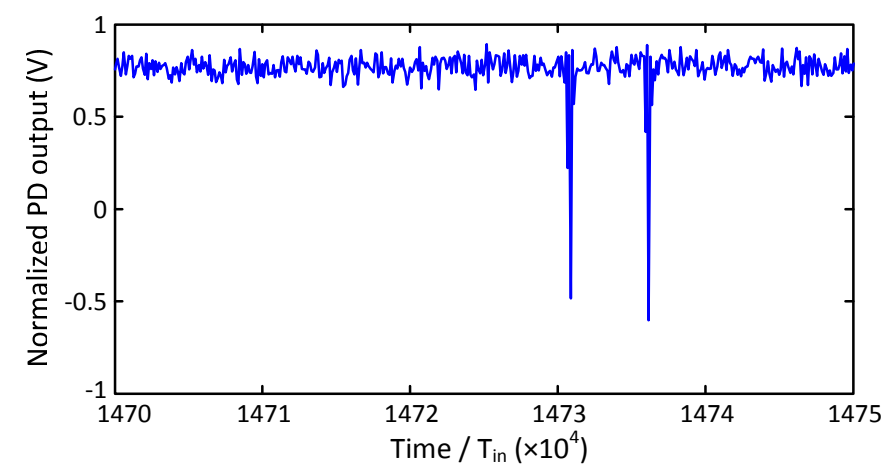

(c)

Fig. 11. Time-domain measurements of the PD output. (a) $\Delta f / \Delta f_{\max }=82 \%$ (b) $\Delta f / \Delta f_{\max }=83 \%$ The number of jumps increases as the frequency shift approaches the border or the synchronization band. (c) Two consecutive jumps of case (b) close in time, showing that the jumps do not repeat periodically.

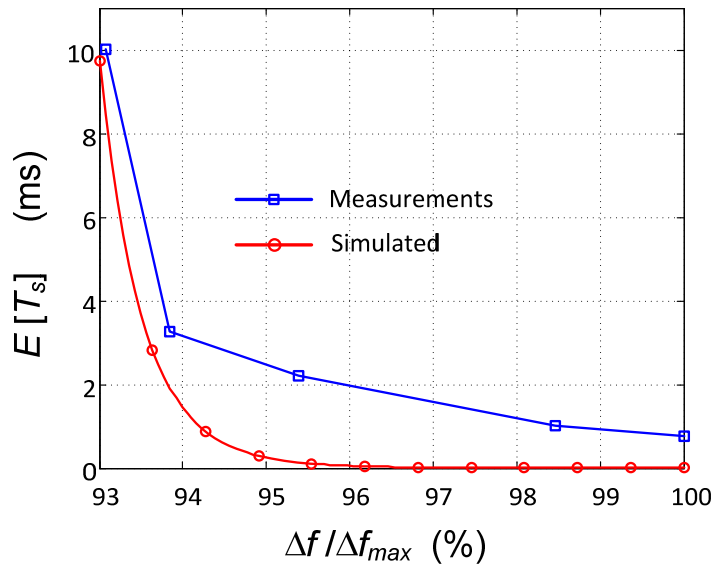

Fig. 12. Measured mean time between jumps. In order to calculate the mean value $E\left[T_{s}\right]$, a group of $N=10$ measurements, corresponding to a time interval of $50 \mathrm{~ms}$ have been obtained. The value $E\left[T_{s}\right]$ increases as the frequency shift is reduced. The results of the stochastic simulation technique have been superimposed.

The evolution of the synchronized solution in the phase space has also been measured. The analysis has been carried out in the FET-based ILO at $5 \mathrm{GHz}$, with the schematic shown in Fig. 1, and with $\Delta f_{\max }=5 \mathrm{MHz}$. In this representation, the ILO state variables $v_{\text {in }}(t)$ and $v_{\text {out }}(t)$ have been chosen, representing the voltages at the ILO input and output nodes, respectively. In Figs. 13(a,b), the points $P_{k}=\left[v_{\text {in }}\left(t_{k}\right), v_{\text {out }}\left(t_{k}\right)\right]$ with $t_{k}=k \Delta t, k=0,1,2, \ldots$ have been represented in the plane $\left(v_{\text {in }}, v_{\text {out }}\right)$. For this analysis, both signals $v_{\text {in }}(t)$ and $v_{\text {out }}(t)$ have been analyzed in the oscilloscope in the $(X-Y)$ mode. The period $T_{\text {in }}$ of the input reference is not a multiple of the sampling step $\Delta t$. As a consequence, in the absence of noise, the points $P_{k}$ fill a closed curve in the plane, or limit cycle. The noise sources affect this figure in two different ways. On the one hand, they give rise to small phase and amplitude perturbations in the state variables, making the points $P_{k}$ shift slightly from the limit cycle. This phenomenon leads to the thick closed curve of Figs. 13(a,b). In the oscilloscope representation, the lightest colors correspond to the most crowded pixels. On the other hand, as the locking frequency is moved from the center to the edge of the synchronization band, random jumps begin to occur. During each jump, the oscillator is unlocked and the system trajectory abandons the limit cycle, producing points $P_{k}$ both outside and inside the thick closed curve (see Fig. 13(a)). As the edge of the synchronization band is approached, the number of random jumps grows, increasing the number of samples outside and inside the cycle. This phenomenon can be observed in Fig. 13(b). Note that, in both measurements, most of the samples are on the noisy limit cycle, since the mean time between jumps $E\left[T_{s}\right]$ is much bigger than the period of the input reference. 


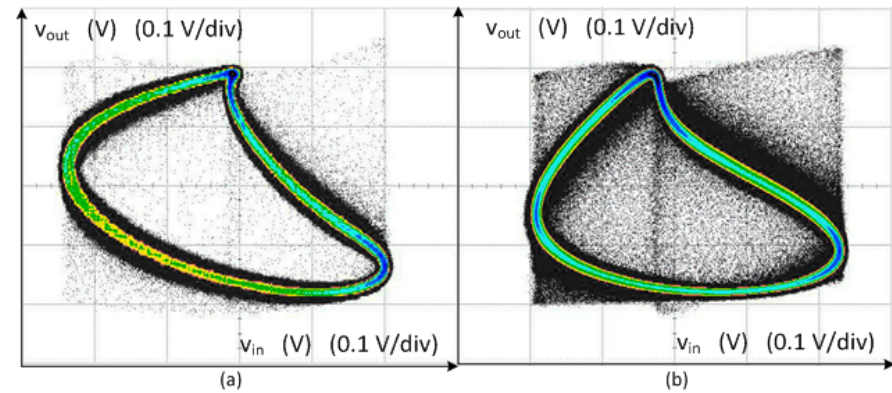

Fig. 13. Time domain measurements. Measuring time $T_{m}=10 \mathrm{~s}$. (a) $\Delta f / \Delta f_{\max }=40 \%$ (b) $\Delta f / \Delta f_{\max }=95 \%$

\section{B. Frequency domain measurements}

Using a measurement set-up based on the reference [33], a frequency domain representation of the injection-locked solution of the oscillator in Fig. 8 has been carried out. This set-up is presented in Fig. 14. Here, an Agilent's N5242A PNA-X Microwave Network Analyzer has been used instead of the setup proposed in [33]. Port 1 of the PNA is used as an injection source to synchronize the oscillator. Fig. 15(a) shows the polar diagram of the parameter $S_{21}$ obtained by sweeping the frequency of the injection port at $P_{i n}=-10 \mathrm{dBm}$. The resulting plot, shown in Fig. 15(a), is in agreement with the injection-locking diagram presented in [33]. The section of the diagram with similar magnitude values corresponds to the injection-locked frequency range, with a phase variation between the two synchronization edges of about $180^{\circ}$ [33]. The remaining two sections, through which the magnitude decays significantly, correspond to frequency intervals for which the oscillator is not synchronized to the input signal. Fig. 15(b) shows the polar diagram obtained by sweeping the injection input power from $P_{i n}=-15 \mathrm{dBm}$ to $10 \mathrm{dBm}$, for a constant frequency value. For the lower range of input power, in the neighborhood of the synchronization edge, an erratic variation of the phase can be observed, though there is a limited capability to capture the cycle slips. In this set-up and that in [33], the detection of the cycle slips is mostly limited by the sweep time required by the PNA (in the order of milliseconds).

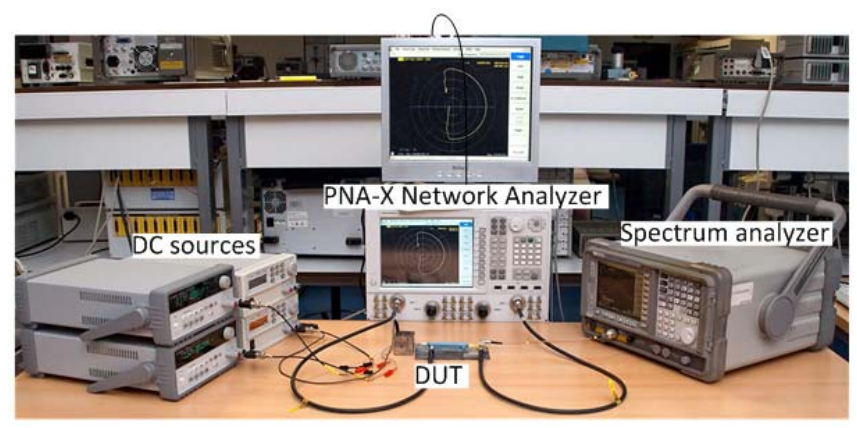

Fig. 14. Frequency domain measurement setup. The measurements were obtained using an Agilent's N5242A PNA-X Microwave Network Analyzer.

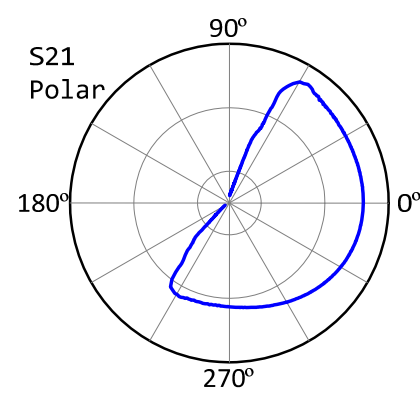

(a)

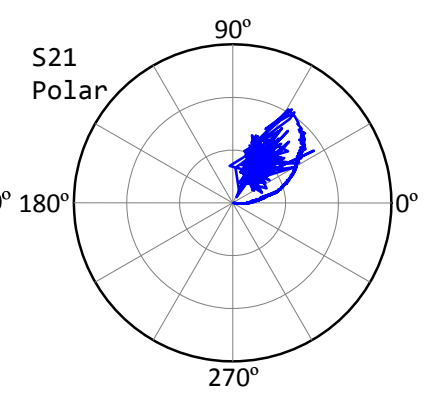

(b)
Fig. 15. Measured polar diagrams of the parameter $S_{21}$. (a) Polar diagram obtained sweeping the injection port frequency. (b) Polar diagram obtained sweeping the input power.

\section{CONCLUSION}

A technique has been presented for the analysis of the cycle slipping phenomenon in fundamentally synchronized oscillators and frequency dividers. It relies on an improved semi-analytical formulation of the injection-locked oscillator, which describes accurately the dependence on the synchronizing source. A detailed analysis of the FokkerPlanck equation associated with this semi-analytical model is carried out using two different resolution methods. Both methods provide expressions for the mean time between jumps in terms of eigenvalues that can be efficiently determined. The technique has enabled the investigation of the influence of the circuit parameters on the probability of random jumps and the possible limitation of the usable synchronization band. The experimental characterization setup allows the measurement of time-domain realizations containing random jumps. In this manner has been possible to characterize experimentally the mean time between phase jumps. It has been applied to a FET-based ILO at $5 \mathrm{GHz}$ (Fig. 1) and to a BJT-based ILO at $1 \mathrm{GHz}$ (Fig. 8) obtaining good agreement with envelope-transient simulations and measurements.

\section{REFERENCES}

[1] H. Meyr and G. Ascheid, Synchronization in Digital Communications, Wiley Inter-Science, New York, 1990

[2] D. Ryter and H. Meyr, "Theory of Phase Tracking Systems of Arbitrary Order: Statistics of Cycle Slips and Probability Distribution of the State Vector," IEEE Transactions on Information Theory, vol. 24, no. 1, January 1978

[3] G. Ascheid and H. Meyr, "Cycle Slips in Phase-Locked Loops: A Tutorial Survey," IEEE Transactions on Communications, vol. 30, no. 10, October 1982

[4] U. L. Rohde, Microwave and Wireless Synthesizers: Theory and Design, Wiley Inter-Science, New York, 1997

[5] J. A. Crawford, Advanced Phase-Lock Techniques, Artech House, Boston, 2008

[6] D. Ryter and P. Talkner, "Rate of Phase slips of a driven Van der Pol Oscillator at low noise," Physics Letters, vol. 93A, no. 9, pp. 447-450, February, 1983

[7] H.R. Rategh and T.H. Lee, "Superharmonic injection-locked frequency dividers", IEEE Journal of Solid-State Circuits, vol. 34, no. 6, pp. 813821, Jun. 1999 
[8] P. Kinget, R. Melville, D. Long and V. Gopinathan, "An injectionlocking scheme for precision quadrature generation," IEEE Journal of Solid-State Circuits, vol 37, no. 7, pp. 845-851, Jul. 2002

[9] C. Van den Bos and C.J.M. Verhoeven, "Frequency division using an injection-locked relaxation oscillator," 2002 IEEE ISCAS International Symposium on Circuits and Systems, May 2002, vol 4. pp. 517-520.

[10] A. Mazzanti, P. Uggetti and F. Svelto, "Analysis and design of injectionlocked LC dividers for quadrature generation," IEEE Journal of SolidState Circuits, vol 39, no. 9, pp. 1425-1433, September 2004.

[11] B. Razavi, "A Study of Injection Locking and Pulling in Oscillators", IEEE Journal of Solid-State Circuits, vol 39, no. 9, pp. 1425-1433, September 2004

[12] A. Suárez, Analysis and Design of Autonomous Microwave Circuits, John Wiley \& Sons, 2009

[13] F. Ramirez, M. Ponton, S. Sancho and A. Suarez, "Phase-Noise Analysis of Injection-Locked Oscillators and Analog Frequency Dividers," IEEE Transactions on Microwave Theory and Techniques, vol. 56, no.2, pp.393-407, Feb. 2008

[14] M. Tiebout, "A CMOS Direct Injection-Locked Oscillator Topology as High-Frequency Low-Power Frequency Divider", IEEE Journal of Solid State Circuits, vol. 39, no, 7, July 2007

[15] S. Drew and V. F. Fusco, "Phase modulated active antenna", Electronics Letters, no.10, pp.835-836, May 1993.

[16] X. Zeng and Z. Chen, "Frequency multiplication and QPSK modulation with subharmonic injection-locked active antenna," Second Annual Conference on Communication Networks and Services Research, 2004. Proceedings, pp.329-332, 19-21 May 2004

[17] L. Dussopt, J. M. Laheurte, A. H. Lettington, P. Papakosta and D. Dunn, "BPSK and QPSK modulations of an oscillating antenna for transponding applications", IEE Proceedings Microwaves, Antennas and Propagation, vol.5, pp. 335-338, October 2000.

[18] G. Wen, F. Xie , P. Ge, J. Li and J. Zhang, "An injection locked oscillator for direction-conversion BPSK modulation applications," ICMMT 4th International Conference on Microwave and Millimeter Wave Technology, 2004.

[19] X. Liu, L. Law Choi, Z. Shen, S. Aditya, C. Qian and Z. Sun, "New approach for QPSK modulation", Vehicular Tech. Conf. 2001, vol.2, pp. 1225-1228, 6-9 May 2001.

[20] J. M. Lopez-Villegas, J. G. Macias, J. A. Osorio, J. Cabanillas, J. J. Sieiro, J. Samitier and N. Vidal, "Continuous phase shift of sinusoidal signals using injection locked oscillators", Microwave and Wireless Components Letters, no.5, pp.312-314, May 2005

[21] L. Zhang and $\mathrm{H}$. Wu, "A double-balanced injection-locked frequency divider for tunable dual-phase signal generation," IEEE Radio Frequency Integrated Circuits (RFIC) Symposium, June 2006, pp. 4

[22] J. Dominguez, A. Suarez and S. Sancho, "Semi-analytical formulation for the analysis and reduction of injection-pulling in front-end oscillators," IEEE Microwave Symposium Digest, pp. 1589-1592, 7-12 June 2009

[23] S. Sancho, A. Súarez and F. Ramírez, "Nonlinear analysis of cycle slips in injection-locked oscillators", IEEE Microwave Symposium Digest, June 2014, pp. 1-3

[24] H. Risken, The Fokker-Planck equation, Springer, New York, 1988

[25] F. X. Kaertner, "Analysis of white and $f-\alpha$ noise in oscillators," Int. Journal of Circuit Theory and Applications, vol. 18, pp. 485-519, 1990.

[26] A. Demir, A. Mehrotra and J. Roychowdhury, "Phase noise in oscillators: a unifying theory and numerical methods for characterization," IEEE Transactions on Circuits and Systems I: Fundamental Theory and Applications, vol. 47, no.5, pp. 655-674, May 2000.

[27] A. Demir and J. Roychowdhury, "A reliable and efficient procedure for oscillator PPV computation, with phase noise macromodeling applications," IEEE Transactions on Computer-Aided Design of Integrated Circuits and Systems, vol. 22, no. 2, pp.188-197, Feb. 2003

[28] K. Kurokawa, "Some basic characteristics of broadband negative resistance oscillators," The Bell System Technical Journal, vol. 48, pp. 1937-1955, July-August. 1969

[29] S. Sancho, A. Suárez, J. Domínguez and F. Ramírez, “Analysis of NearCarrier Phase-Noise Spectrum in Free-Running Oscillators in the
Presence of White and Colored Noise Sources," IEEE Transactions on Microwave Theory and Techniques, vol. 58, no. 3, 2010, pp. 587 - 601

[30] A. A. Andronov and L. S. Pontryagin, Geometric methods in the theory of differential equations, Springer-Verlag, New York, 1988

[31] W.E. Boyce and R. C. DiPrima, Elementary Differential Equations and Boundary Value Problems, John Wiley \& Sons, 2005

[32] R. Courant and D. Hilbert, Methods of Mathematical Physics, John Wiley \& Sons, New York, 1989

[33] A. P. S. Khanna and J. Obregon, "Direct Measurement of the Nonlinear M. I. C. Oscillator Characteristics Using Injection Locking Polar Diagram," IEEE MTT-S International Microwave Symposium Digest, pp. 501-503, May 31-June 3, 1983

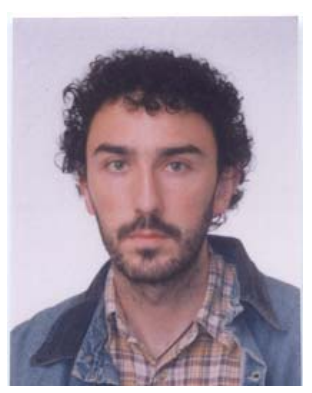

Sergio Sancho (A'04-M'04) was born in Santurce, Spain, in 1973. In 1997 received the degree in Physics from Basque Country University. In 1998 he joined the Communications Engineering Department of the University of Cantabria, Spain, where he received the Ph.D. degree in Electronic Engineering in February 2002. At present, he works at the University of Cantabria, as an Associate Professor of its Communications Engineering Department. His research interests include the nonlinear analysis of microwave autonomous circuits and frequency synthesizers, including stochastic and phase-noise analysis.

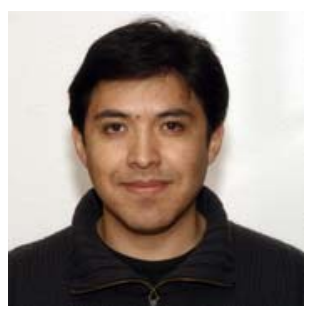

Franco Ramírez (S'03-A'05-M'05) was born in Potosí, Bolivia. He obtained a degree in electronic systems engineering degree from the Military School of Engineering (EMI) in La Paz, Bolivia, in 2000 and the Ph.D. degree in communications engineering from the University of Cantabria, Santander, Spain in 2005. From 1999 to 2000 he worked for Ericsson de Bolivia Telecomunicaciones, where he was involved in several projects related with GSM and TDMA technologies. At present he is a Research Associate, under the "Ramón y Cajal" Programme of the Spanish Ministry of Science and Innovation, at the Communications Engineering Department of the University of Cantabria. His research interests include phase noise, stability and the development of nonlinear techniques for the analysis and design of autonomous microwave circuits.

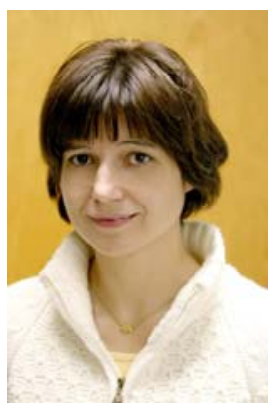

Almudena Suárez (M'96-SM’01-F'12) was born in Santander, Spain. She received the degree in electronic physics and the Ph.D. degree from the University of Cantabria, Santander, Spain, in 1987 and 1992, respectively, and the Ph.D. degree in electronics from the University of Limoges, Limoges, France, in 1993. At present, she is a Full Professor at the University of Cantabria, and a member of its Communications Engineering Department. She has authored the book Analysis and design of autonomous microwave circuits for the publisher IEEE-Wiley and co-authored the book Stability analysis of microwave circuits for the publisher Artech-House. She belongs to the technical committees of the IEEE International Microwave Symposium and European Microwave Conference. She was an IEEE Distinguished Microwave Lecturer for the period 2006-2008. She is the coordinator of the research area "Electronic and Communications Technology" of the Spanish national evaluation agency ANEP. 